\title{
Dereplication and Quantification of Major Compounds of Convolvulus arvensis L. Extracts and Assessment of Their Effect on LPS-Activated J774 Macrophages
}

\author{
Hafiz Abdul Khaliq 1,2,3 ${ }^{\mathbb{D}}$, Sergio Ortiz ${ }^{1}$ (D), Mireille Alhouayek ${ }^{2}$, Giulio G. Muccioli ${ }^{2}$ (D) \\ and Joëlle Quetin-Leclercq ${ }^{1, * \mathbb{D}}$
}

check for updates

Citation: Abdul Khaliq, H.; Ortiz, S.; Alhouayek, M.; Muccioli, G.G.; Quetin-Leclercq, J. Dereplication and Quantification of Major Compounds of Convolvulus arvensis L. Extracts and Assessment of Their Effect on LPS-Activated J774 Macrophages. Molecules 2022, 27, 963.

https://doi.org/10.3390/

molecules 27030963

Academic Editors: Maria Fernanda Taviano, Rosaria Acquaviva and Giuseppe Antonio Malfa

Received: 30 November 2021

Accepted: 27 January 2022

Published: 31 January 2022

Publisher's Note: MDPI stays neutral with regard to jurisdictional claims in published maps and institutional affiliations.

Copyright: (C) 2022 by the authors. Licensee MDPI, Basel, Switzerland. This article is an open access article distributed under the terms and conditions of the Creative Commons Attribution (CC BY) license (https:// creativecommons.org/licenses/by/ $4.0 /)$.
1 Pharmacognosy Research Group, Louvain Drug Research Institute, UCLouvain, 1200 Brussels, Belgium; hafiz.abdulkhaliq@uclouvain.be (H.A.K.); sergio.ortiz@uclouvain.be (S.O.)

2 Bioanalysis and Pharmacology of Bioactive Lipids Research Group, Louvain Drug Research Institute, UCLouvain, 1200 Brussels, Belgium; mireille.alhouayek@uclouvain.be (M.A.); giulio.muccioli@uclouvain.be (G.G.M.)

3 Department of Pharmacognosy, Faculty of Pharmacy, Bahauddin Zakariya University, Multan 60800, Pakistan * Correspondence: joelle.leclercq@uclouvain.be; Tel.: +32-2-764-72-54

\begin{abstract}
Convolvulus arvensis is used in Pakistani traditional medicine to treat inflammation-related disorders. Its anti-inflammatory potential was evaluated on hexane, dichloromethane, ethyl acetate, methanol, and aqueous extracts of whole plant on pro-inflammatory mediators in LPS-activated murine macrophage J774 cells at the non-cytotoxic concentration of $50 \mu \mathrm{g} / \mathrm{mL}$. Ethyl acetate (ARE) and methanol (ARM) extracts significantly decreased mRNA levels of IL-6, TNF- $\alpha$, MCP-1, COX-2, and iNOS. Furthermore, both extracts dose dependently decreased IL- 6 , TNF- $\alpha$, and MCP- 1 secretion. Forty-five compounds were putatively identified in ARE and ARM by dereplication (using HPLC-UVHRMS $^{n}$ analysis and molecular networking), most of them are reported for the first time in C. arvensis, as for example, nineteen phenolic derivatives. Rutin, kaempferol-3-O-rutinoside, chlorogenic acid, 3,5di-O-caffeoylquinic acid, $\mathrm{N}$-trans- $p$-coumaroyl-tyramine, and $\mathrm{N}$-trans-feruloyl-tyramine were main constituents identified and quantified by HPLC-PDA in ARE and ARM. Furthermore, chlorogenic acid, tyramine derivatives, and the mixture of the six identified major compounds significantly decreased IL-6 secretion by LPS-activated J774 cells. The activity of $N$-trans- $p$-coumaroyl-tyramine is shown here for the first time. Our results indicate that ARE, ARM and major constituents significantly inhibited the expression of pro-inflammatory mediators, which supports the use of this plant to treat inflammatory diseases.
\end{abstract}

Keywords: field bindweed; GNPS; molecular networking; HPLC-UV-HRMS; phenolics; inflammation; biological screening

\section{Introduction}

Convolvulus arvensis L. belonging to the family Convolvulaceae is among the very frequently used plants in the traditional medicine of Pakistan to treat inflammatory conditions [1]. C. arvensis is a perennial deep-rooted creeping weed found in temperate regions throughout the world. Its common English name is "field bindweed", while in Pakistan it is locally called "leli" or "wanveri" [2,3].

In the traditional medicine of Pakistan, roots of C. arvensis are used as purgatives [4], while the leaves' paste is applied topically to treat boils, inflammation, and rheumatism [3,5,6]. To cure constipation, dried whole plant mixed with molasses is given with milk at night or fresh whole plant boiled in water is eaten as a vegetable with wheat bread [6,7]. Fresh plant ground with black peppers in water is given to treat bleeding piles, leprosy, and other skin diseases [3,6]. C. arvensis is reported to possess antioxidant [8], antiarthritic [9,10], hepatoprotective [11], and hypoglycemic [12] activities. Phytochemical investigation has shown 
the presence of tropane alkaloids [13], coumarins [12], resin glycosides [14], flavonoids [15], phenolic acids [8], and phytosterols [16] in C. arvensis.

Inflammation is a body's natural defense system triggered by a variety of harmful stimuli such as damaged cells, pathogens, lipopolysaccharides, or irritants. Immune cells, especially macrophages, release biochemical mediators, such as pro-inflammatory cytokines (e.g., IL-6 and TNF- $\alpha$ ) and chemokines (e.g., MCP-1), to coordinate the immune reaction that will eliminate the inflammatory triggers and promote tissue repair and recovery [17]. Likewise, cyclooxygenase 2 (COX-2) and inducible nitric oxide synthase (iNOS) are two important enzymes involved in the inflammatory process [18,19]. However, uncontrolled inflammation may lead to severe disorders such as inflammatory bowel diseases, asthma, rheumatoid arthritis, and neurodegenerative diseases [20].

Traditional medicines are a rich source of new drugs [21]. The phytochemicals that could be responsible for the biological activity of plants in traditional medicine need to be identified and quantified, notably to prepare standardized crude extracts or to isolate them to develop into a new drug. To avoid the isolation of already known compounds, "dereplication" is now extensity used. MS along with the Global Natural Product Social Molecular Networking (GNPS) (http:/ / gnps.ucsd.edu) is getting popular to dereplicate known compounds of natural products [22].

Due to the very frequent use of $C$. arvensis in Pakistani traditional medicine to treat inflammatory conditions, we evaluated this plant and we report the inhibitory effect of $C$. arvensis' extracts on the expression of pro-inflammatory markers in LPS-stimulated J774 macrophage. Furthermore, we describe the dereplication of the major most active crude extracts chemical constituents performed by HPLC-MS/MS and molecular networking. Finally, we report the quantification of their major constituents by HPLC-PDA.

\section{Results and Discussion}

\subsection{Extraction Yield and MTT Assay of Crude Extracts}

We prepared four crude extracts from $C$. arvensis by successive Soxhlet extraction with four solvents of increasing polarity, namely hexane, dichloromethane, ethyl acetate, and methanol (ARH, ARE, ARD, and ARM, respectively), and a decoction (ARW). The purpose of using these solvents was to extract as much chemical constituents as possible and to divide the chemical constituents in four parts depending on their solubility: non-polar to polar, from hexane to methanol. In addition, a decoction was prepared to dissolve the watersoluble constituents and also because, in general, traditional use of plant-based therapies involves their preparation in water, mostly as decoctions. Extraction yields of ARH, ARD, ARE, ARM, and ARW were $1.84 \%, 2.28 \%, 0.66 \%, 9.86 \%$, and $14.31 \%$, respectively. The highest yield of ARW indicates the presence of a high quantity of polar compounds in $C$. arvensis, probably primary metabolites, which are soluble in water. Yields of ARH, ARD, and ARE are very low, compared to ARM, indicating the lower presence of non-polar (fatty) and less polar (e.g., terpenoids) substances. Methanol is a very good solvent for extraction because it can extract both hydrophilic, but also moderately lipophilic substances that are present in higher quantities in this plant. This could explain the relatively high yield of ARM.

The effect of these five extracts on cellular metabolic activity, as a proxy of cytotoxicity, was measured using an MTT assay on two cell lines, namely WI38 and J774. As cytotoxicity varies according to the type of cells, we decided to test two cell lines: J774, the line used for the tests on cytokines production and WI38, to verify that the eventual effects observed on J774 were not specific to this cell line. As frequently performed with crude extracts, two concentrations of these extracts were used, $100 \mu \mathrm{g} / \mathrm{mL}$ and $50 \mu \mathrm{g} / \mathrm{mL}$. Camptothecin was used as positive control. The $\mathrm{IC}_{50}$ values obtained for camptothecin were $34.2 \pm 4.9 \mathrm{ng} / \mathrm{mL}$ and $7.1 \pm 0.6 \mathrm{ng} / \mathrm{mL}$ for WI38 and J774 cells, respectively. When tested at $100 \mu \mathrm{g} / \mathrm{mL}$, most of the extracts decreased the MTT reduction into formazan in both cell lines (Figure 1). At $50 \mu \mathrm{g} / \mathrm{mL}$ concentration, ARH, ARD, ARE, ARM, and ARW showed percentage viability of $3.6 \pm 0.2,5.1 \pm 0.1,98.1 \pm 12.7,94.1 \pm 0.8$, and $108.4 \pm 4.7$ for WI38 cells, and $3.7 \pm 0.1$, 
$6.0 \pm 0.5,77.5 \pm 6.8,92.7 \pm 0.4$, and $97.3 \pm 1.6$ for J774 cells, respectively. The difference in cytotoxicity of the different crude extracts is explained by the presence of different types of compounds in these extracts. ARE, ARM, and ARW, non-cytotoxic at the tested concentrations, were selected to evaluate their effect on LPS-stimulated J744 cells.

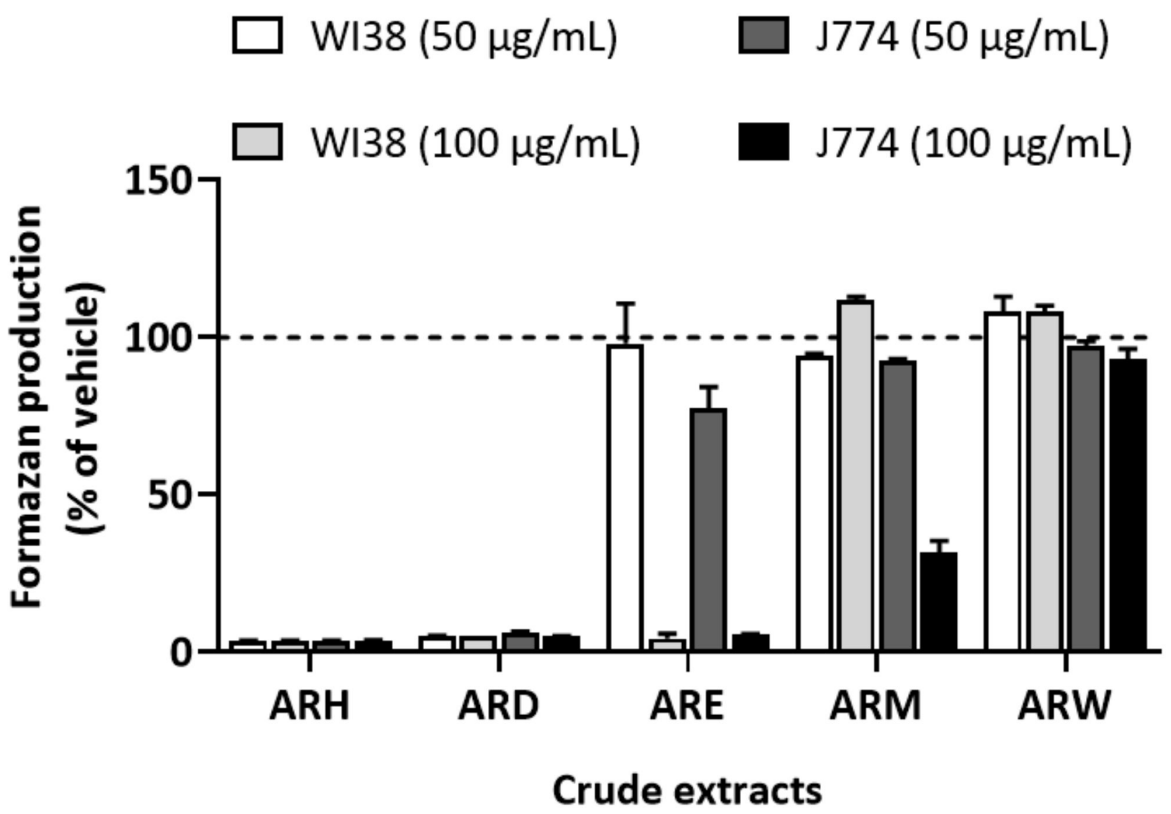

Figure 1. Effect of the crude extracts on cell viability. WI38 and J774 cells were treated with 50 and $100 \mu \mathrm{g} / \mathrm{mL}$ of the crude extracts or vehicle (DMSO and EtOH-H ${ }_{2} \mathrm{O}(25: 75)$ ) for $72 \mathrm{~h}$. Then, an MTT assay was performed. Data are expressed as mean \pm SEM. $n=2$ in triplicates.

\subsection{Effect of ARE, ARM, and ARW on the Expression of Pro-Inflammatory Mediators in} LPS-Stimulated 744 Cells

The effect of ARE, ARM, and ARW was analyzed on LPS-induced J774 cell activation by assessing the expression of cytokines (IL-6, TNF $\alpha$, and MCP-1), COX-2, and iNOS following the incubation of cells with the crude extracts $(50 \mu \mathrm{g} / \mathrm{mL})$ and LPS $(100 \mathrm{ng} / \mathrm{mL})$ for $8 \mathrm{~h}$. IL-6 and TNF- $\alpha$ are not only key pro-inflammatory cytokines of innate immune response, but can also amplify the inflammation to a chronic state. IL-6 plays a key role in regulating Th17 cells (Th17) and regulatory T cells (Treg) response, the former cell type leading to autoimmune disorders while the latter cell type counters its effects [23]. TNF- $\alpha$ augments the transcription of other pro-inflammatory cytokines [24], while MCP1, also referred to as CC chemokine ligand 2 (CCL2), is a chemokine favoring monocytes recruitment [25]. COX-2 is an enzyme that mediates the synthesis of prostaglandins, which are considered as important mediators of the innate immune response [26]. iNOS causes increased production of NO, leading to oxidative stress, tissue damage, and inflammation [19]. First mRNA levels of IL-6 were analyzed by RT-qPCR. ARW did not decrease mRNA levels of IL-6 (data not shown here), while ARE and ARM significantly inhibited the LPS-induced expression of IL-6 mRNA (Figure 2A). The effect of ARE and ARM was further analyzed on TNF- $\alpha$, MCP-1, COX-2, and iNOS mRNA expression. ARE and ARM significantly inhibited TNF- $\alpha$, MCP-1, COX-2, and iNOS expression (Figure 2B-E). To confirm the protein level the effects of ARE and ARM, IL-6, TNF- $\alpha$, and MCP-1 levels in the culture medium were measured as well. Both ARE and ARM dose-dependently decreased LPS-induced IL-6, TNF- $\alpha$, and MCP-1 secretion by J774 cells, further supporting their interesting properties (Figure 2F-H). 

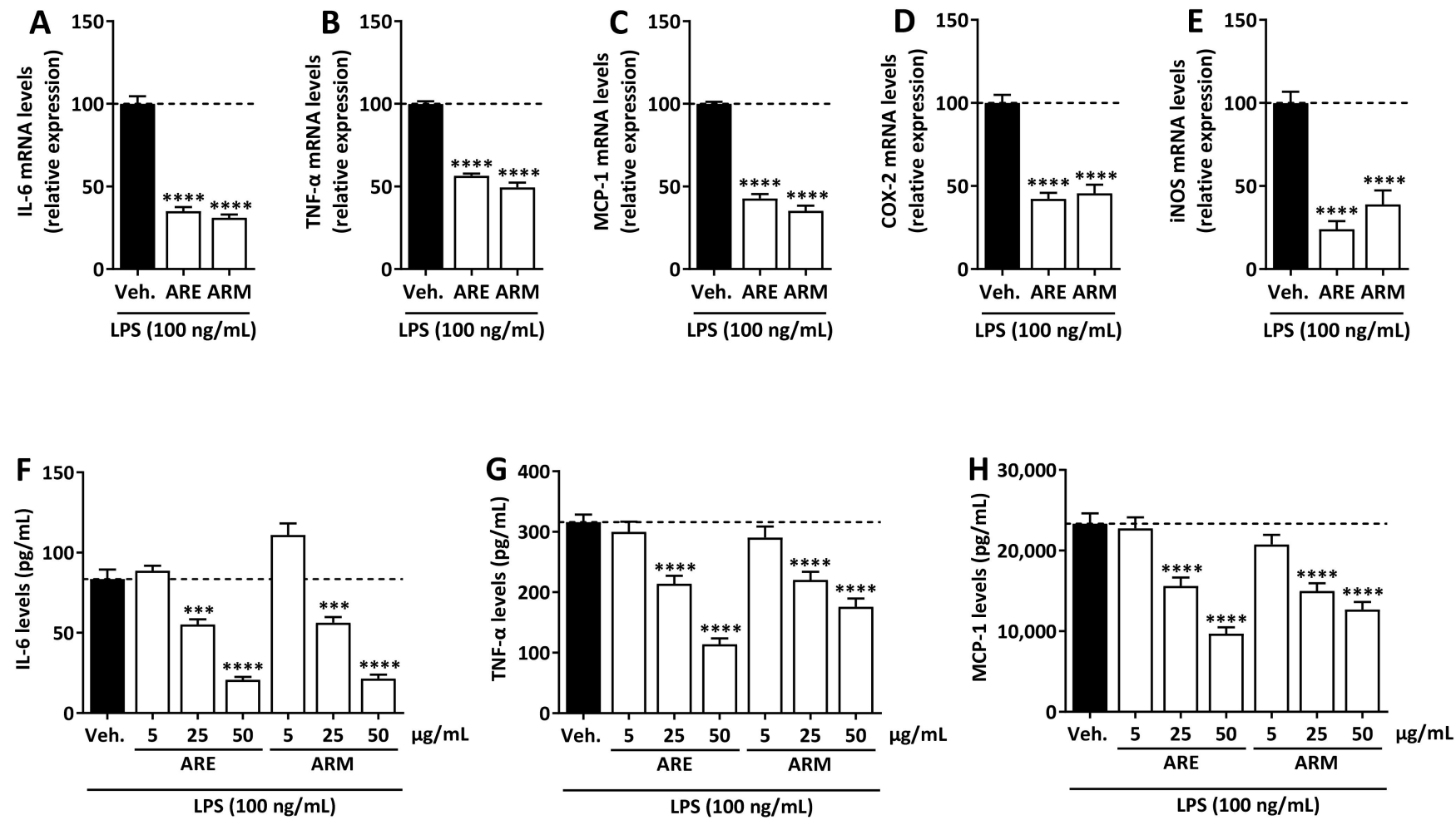

Figure 2. Effect of ARE and ARM on the expression of pro-inflammatory mediators in LPSstimulated J744 cells. (A-E) Cells were incubated with crude extracts (50 $\mu \mathrm{g} / \mathrm{mL}$ ), or vehicle (Veh., $0.25 \%$ DMSO) and LPS (100 ng/mL) for $8 \mathrm{~h}$. mRNA levels of IL-6, TNF- $\alpha$, MCP-1, COX-2, and iNOS were analyzed by RT-qPCR with RPL19 used as reference gene. Results are expressed in percentage of the LPS-vehicle condition. (F-H) Cells were incubated with crude extracts, or vehicle (Veh., $0.25 \%$ DMSO) and LPS (100 ng/mL) for $8 \mathrm{~h}$. IL-6, TNF- $\alpha$, and MCP-1 protein levels in the supernatant medium were quantified by ELISA. IL- 6 , TNF- $\alpha$, and MCP-1 were not detected in the medium of the unstimulated, vehicle-treated cells. The data were analyzed by the one-way ANOVA followed by Dunnet's post hoc test for comparisons between groups, are expressed as mean \pm SEM. $n=3$ in triplicates, ${ }^{* * *} p<0.001,{ }^{* * * *} p<0.0001$ vs. Veh.

\subsection{Dereplication of ARE and ARM}

In order to start exploring the compounds that could potentially mediate the observed effects, we aimed at dereplicating ARE and ARM extracts by HPLC-MS/MS along with the Global Natural Product Social Molecular Networking (GNPS). Previous phytochemical studies have shown that $C$. arvensis contains alkaloids, phenolic acids, flavonoids, sterols, resin glycosides, coumarins, and triterpenes [27]. Based on our LC-UV-MS ${ }^{\text {chromato- }}$ graphic analysis of ARE and ARM, mainly developed to analyze phenolic compounds, which are known to be good anti-inflammatory candidates [28], the main detected metabolites can be organized in three metabolites groups: phenolic acids, flavonoid glycosides, and glycolipids (Table 1). The molecular network of both extracts organizes the fragmented compounds in several clusters, corresponding to phenolics/glycosidic compounds (Figure 3A,B), lipid acids (Figure 3C), triglycosides flavonoids (Figure 3D), and glycoside lipids (Figure 3E). After several sugars, derivatives and small organic molecules, the first eluted phenolic acid was putatively identified as $O$-glucosyl-caffeic acid (10), which gave pseudo-molecular ion $[\mathrm{M}-\mathrm{H}]^{-}$at $m / z 341$ and fragmented to the corresponding aglycon, which gave a signal at $m / z 179$. The same fragment was observed for the phenolic acids identified as chlorogenic acid (3-O-caffeoylquinic acid) (13), O-caffeoylquinic acid (16), 3,4di-O-caffeoylquinic acid (38), and 3,5-di-O-caffeoylquinic acid (41), corresponding to the caffeic acid part of the molecule. Discrimination between the isomers of di-O-caffeoylquinic acids was based on their $\mathrm{MS}^{2}$ fragmentation spectra, a base signal at $m / z 173$ for 4-acyl 
derivatives, at $m / z 191$ for 5-acyl derivatives, and at $m / z 179$ for 3-acyl derivatives, depending on the proton transfer, as previously reported [29]. 4,5-Di-O-caffeoylquinic acid (43) was also identified in the same way with base signal at $m / z 173$. The presence of the pseudo-molecular ion $[\mathrm{M}-\mathrm{H}]^{-}$at $m / z 179$ also allowed us to identify caffeic acid (24). Feruloyl-quinic acid derivatives were also detected with a pseudo-molecular ion $[\mathrm{M}-\mathrm{H}]^{-}$at $\mathrm{m} / z 367$ for $O$-feruloyl-quinic acid (26) and at $m / z 529$ for caffeoyl-feruloylquinic acid derivatives $(46,47)$. The flavonoids glycosides putatively identified were mostly various quercetin and kaempferol derivatives. Quercetin-O-pentosyl-hexosyl-hexoside $\left(25[\mathrm{M}-\mathrm{H}]^{-} \text {at } m / z \text { 741, [M-pentosyl }-\mathrm{H}\right]^{-}$at $\left.m / z 609\right)$, rutin $\left(29,[\mathrm{M}-\mathrm{H}]^{-}\right.$at $m / z$ 609) and quercetin-O-(hydroxy-3-methylglutaryl)-hexoside (36, [M - $\mathrm{H}]^{-}$at $\mathrm{m} / \mathrm{z} 607$, [Mhydroxy-3-methylglutaryl $-\mathrm{H}]^{-}$at $m / z$ 463) presented the characteristic fragment signal at $m / z 301 / 300$, corresponding to the quercetin aglycon (with a loss of one or two $\mathrm{H}$ ) [30]. Moreover, kaempferol-O-pentosyl-hexosyl-hexoside (28, [M- H] ${ }^{-}$at $m / z$ 725, [M-pentosyl $-\mathrm{H}]^{-}$at $m / z$ 593), kaempferol-O-hexosyl-pentoside (33, [M - H] ${ }^{-}$at $m / z$ 593), kaempferol3-O-rutinoside (35, $[\mathrm{M}-\mathrm{H}]^{-}$at $m / z$ 593), kaempferol-O-hexoside $\left(37,[\mathrm{M}-\mathrm{H}]^{-}\right.$at $m / z$ 447), kaempferol-O-pentoside (42, $[\mathrm{M}-\mathrm{H}]^{-}$at $m / z$ 417) were identified as kaempferol derivatives by the presence of the important signal at $m / z 284 / 285$, corresponding to the kaempferol aglycone (with a loss of one or two H). Glycolipid derivatives, mainly detected in ARE, were also putatively identified as two trihydroxy-dienoic acid derivatives (51 and 52, [M - $\mathrm{H}]^{-}$at $m / z$ 327) and a trihydroxy-octadecenoic acid derivative (53, $[\mathrm{M}-\mathrm{H}]^{-}$at $m / z$ 329). Four hexosyl lipids were also possibly identified as $O$-(hexosylhexosyl)-O-linolenoyl-glycerol (55, [M - $\mathrm{H}]^{-}$at $m / z$ 675, [M-linolenoyl $\left.-\mathrm{H}\right]^{-}$at $m / z$ 397), O-hexosyl-O-linolenoyl-glycerol (57, [M - $\mathrm{H}]^{-}$at $m / z$ 559, [M-hexosyl-glycerol $\left.-\mathrm{H}\right]^{-}$ at $m / z$ 277), O-(hexosyl-hexosyl)-O-palmitoyl-glycerol $\left(58,[\mathrm{M}-\mathrm{H}]^{-}\right.$at $m / z$ 699, [Mpalmitoyl $-\mathrm{H}^{-}$at $m / z$ 397) and O-hexosyl-di-O-linolenoyl-glycerol $\left(\mathbf{6 0},[\mathrm{M}-\mathrm{H}]^{-}\right.$at $m / z$ 559, [M-O-linolenoyl-O-hexosyl-glycerol $-\mathrm{H}]^{-}$at $m / z$ 277). In addition, the presence of two tyramines was also proposed: $N$-trans-p-coumaroyl-tyramine $\left(48,[\mathrm{M}-\mathrm{H}]^{-}\right.$at $m / z 282$, and a fragment corresponding to coumaramide at $m / z$ 162) and $N$-trans-feruloyl-tyramine (49, $[\mathrm{M}-\mathrm{H}]^{-}$at $m / z 312$, and a fragment corresponding to [ferulamide- $\left.\mathrm{CH}_{2}-\mathrm{H}\right]^{-}$at $m / z 178)$, mainly detected in ARE.

Table 1. Putative identification of chemical constituents present in C. arvensis extracts (ARE and ARM) by comparison of the $\mathrm{MS}^{2}$ data (Negative mode, APCI).

\begin{tabular}{|c|c|c|c|c|c|c|c|c|c|c|c|}
\hline Code & $\begin{array}{c}t_{R} \\
{[\mathrm{~min}]}\end{array}$ & $\lambda_{\max }$ & $\mathrm{m} / \mathrm{z}$ & MS Major Ion(s) & $\begin{array}{c}\text { MS/MS } \\
\text { Fragments } \\
{[m / z]}\end{array}$ & $\begin{array}{l}\text { Molecular } \\
\text { Formula }\end{array}$ & $\begin{array}{c}\Delta \\
\text { ppm }\end{array}$ & $\underset{\mathrm{mDa}}{\Delta}$ & Putative Identification & $\begin{array}{c}\text { Isolated } \\
\text { Previously }^{\mathrm{a}}\end{array}$ & Ref. \\
\hline 1 & 3.20 & n.d. & $\begin{array}{l}387.1147 \\
341.1084 \\
729.2287 \\
683.2222\end{array}$ & $\begin{array}{c}{\left[\mathrm{M}+\mathrm{HCOO}^{-}\right]^{-}} \\
{[\mathrm{M}-\mathrm{H}]^{-}} \\
{\left[2 \mathrm{M}+\mathrm{HCOO}^{-}\right]^{-}} \\
{[2 \mathrm{M}-\mathrm{H}]^{-}}\end{array}$ & $179.0563^{\mathrm{b}}$ & $\mathrm{C}_{12} \mathrm{H}_{22} \mathrm{O}_{11}$ & 0.04 & 0.01 & Sucrose & & \\
\hline 2 & 3.28 & n.d. & $\begin{array}{l}151.0613 \\
197.0664\end{array}$ & $\begin{array}{c}{[\mathrm{M}-\mathrm{H}]^{-}} \\
{\left[\mathrm{M}+\mathrm{HCOO}^{-}\right]^{-}}\end{array}$ & $\begin{array}{c}71.0142 ; \\
101.0247 ; \\
133.0509^{\mathrm{b}} \\
\end{array}$ & $\mathrm{C}_{5} \mathrm{H}_{12} \mathrm{O}_{5}$ & 4.31 & 0.65 & Xylitol $^{\#}$ & & \\
\hline 3 & 3.40 & n.d. & $\begin{array}{l}181.0722 \\
135.0668\end{array}$ & $\begin{array}{c}{\left[\mathrm{M}+\mathrm{HCOO}^{-}\right]^{-}} \\
{[\mathrm{M}-\mathrm{H}]^{-}}\end{array}$ & $135.0669^{c}$ & $\mathrm{C}_{5} \mathrm{H}_{12} \mathrm{O}_{4}$ & -2.01 & -0.27 & $\begin{array}{l}\text { 2-methyl-1,2,3,4- } \\
\text { butanetetrol }\end{array}$ & & \\
\hline 4 & 3.39 & 270 & $\begin{array}{l}191.0567 \\
383.1199\end{array}$ & $\begin{array}{c}{[\mathrm{M}-\mathrm{H}]^{-}} \\
{[2 \mathrm{M}-\mathrm{H}]^{-}} \\
\end{array}$ & $\begin{array}{l}85.0299 ; \\
127.0405 \\
173.0458 ; \\
93.0350^{\mathrm{b}}\end{array}$ & $\mathrm{C}_{7} \mathrm{H}_{12} \mathrm{O}_{6}$ & 5.95 & 1.14 & Quinic acid ${ }^{\#}$ & C. althaeoides & [31] \\
\hline 5 & 3.59 & n.d. & $\begin{array}{l}239.0775 \\
193.0723 \\
\end{array}$ & $\begin{array}{c}{\left[\mathrm{M}+\mathrm{HCOO}^{-}\right]^{-}} \\
{[\mathrm{M}-\mathrm{H}]^{-}}\end{array}$ & $\begin{array}{l}133.0509 \\
59.0141^{\mathrm{b}}\end{array}$ & $\mathrm{C}_{7} \mathrm{H}_{14} \mathrm{O}_{6}$ & 5.93 & 1.09 & $\begin{array}{c}\text { O-methyl-inositol } \\
\text { isomer I }\end{array}$ & & \\
\hline 6 & 3.96 & n.d. & 239.0772 & {$\left[\mathrm{M}+\mathrm{HCOO}^{-}\right.$} & $\begin{array}{l}193.0718 ; \\
133.0508 ; \\
59.0141^{\mathrm{b}}\end{array}$ & $\mathrm{C}_{7} \mathrm{H}_{14} \mathrm{O}_{6}$ & 2.12 & 0.51 & $\begin{array}{l}\text { O-methyl-inositol } \\
\text { isomer II }\end{array}$ & & \\
\hline
\end{tabular}


Table 1. Cont.

\begin{tabular}{|c|c|c|c|c|c|c|c|c|c|c|c|}
\hline Code & $\underset{[\mathrm{min}]}{t_{\mathrm{R}}}$ & $\lambda_{\max }$ & $\mathrm{m} / \mathrm{z}$ & MS Major Ion(s) & $\begin{array}{c}\text { MS/MS } \\
\text { Fragments } \\
{[\mathrm{m} / \mathrm{z}]}\end{array}$ & $\begin{array}{l}\text { Molecular } \\
\text { Formula }\end{array}$ & $\begin{array}{c}\Delta \\
\text { ppm }\end{array}$ & $\underset{\mathrm{mDa}}{\Delta}$ & Putative Identification & $\begin{array}{c}\text { Isolated } \\
\text { Previously }\end{array}$ & Ref. \\
\hline 7 & 4.90 & n.d. & $\begin{array}{l}239.0772 \\
193.0718 \\
712.5355\end{array}$ & $\begin{array}{c}\left.\mathrm{M}+\mathrm{HCOO}^{-}\right]^{-} \\
{[\mathrm{M}-\mathrm{H}]^{-}} \\
{[\mathrm{M}-\mathrm{H}]^{-}}\end{array}$ & $\begin{array}{l}133.0508 ; \\
59.0141^{\mathrm{b}}\end{array}$ & $\mathrm{C}_{7} \mathrm{H}_{14} \mathrm{O}_{6}$ & 3.04 & 0.59 & $\begin{array}{l}\text { O-methyl-inositol } \\
\text { isomer III }^{\#}\end{array}$ & & \\
\hline 8 & 4.98 & n.d. & 117.0198 & {$[\mathrm{M}-\mathrm{H}]^{-}$} & $\begin{array}{l}73.0297 \\
99.0091^{\mathrm{b}}\end{array}$ & $\mathrm{C}_{4} \mathrm{H}_{6} \mathrm{O}_{4}$ & 8.69 & 1.02 & Succinic acid $\#$ & & \\
\hline 9 & 5.06 & n.d. & 281.0881 & {$[\mathrm{M}-\mathrm{H}]^{-}$} & $\begin{array}{l}235.0820 \\
263.0955^{\text {b }}\end{array}$ & $\mathrm{C}_{10} \mathrm{H}_{18} \mathrm{O}_{9}$ & 3.00 & 0.84 & Xylobiose $^{\#}$ & & \\
\hline 10 & 5.68 & n.d. & 341.0889 & {$[\mathrm{M}-\mathrm{H}]^{-}$} & $\begin{array}{r}179.0348 ; \\
167.0349 \\
135.0451^{\mathrm{b}} \\
\end{array}$ & $\mathrm{C}_{15} \mathrm{H}_{18} \mathrm{O}_{9}$ & 4.82 & 1.64 & $\begin{array}{c}O \text {-glucosyl-caffeic acid } \\
\text { isomer }\end{array}$ & & \\
\hline 11 & 6.02 & n.d. & $\begin{array}{l}451.2199 \\
405.2110\end{array}$ & $\begin{array}{c}{\left[\mathrm{M}+\mathrm{HCOO}^{-}\right]^{-}} \\
{[\mathrm{M}-\mathrm{H}]^{-}}\end{array}$ & $\begin{array}{l}167.1076 ; \\
179.0560 ; \\
243.1596^{\mathrm{b}}\end{array}$ & $\mathrm{C}_{19} \mathrm{H}_{34} \mathrm{O}_{9}$ & -3.60 & -1.46 & $\begin{array}{c}\text { Magastigmane } \\
\text { glycoside derivative I }\end{array}$ & & \\
\hline 12 & 6.18 & $\begin{array}{l}289 \\
325\end{array}$ & 433.2091 & {$\left[\mathrm{M}+\mathrm{HCOO}^{-}\right]^{-}$} & $\begin{array}{r}387.2012 ; \\
179.0559 ; \\
161.0454^{\mathrm{c}} \\
\end{array}$ & $\mathrm{C}_{19} \mathrm{H}_{32} \mathrm{O}_{8}$ & 3.99 & 1.73 & $\begin{array}{c}\text { Magastigmane } \\
\text { glycoside derivative II \# }\end{array}$ & & \\
\hline 13 & 6.21 & n.d. & 353.0881 & {$[\mathrm{M}-\mathrm{H}]^{-}$} & $\begin{array}{l}191.0559 ; \\
179.0350 ; \\
173.0455 ; \\
135.0452^{\mathrm{b}}\end{array}$ & $\mathrm{C}_{16} \mathrm{H}_{18} \mathrm{O}_{9}$ & 2.39 & 0.84 & Chlorogenic acid * & $\begin{array}{l}\text { C. arvensis; } \\
\text { C. dorycnium }\end{array}$ & {$[32,33]$} \\
\hline 14 & 7.30 & 315 & $\begin{array}{l}369.0820 \\
323.0769 \\
\end{array}$ & $\begin{array}{c}{\left[\mathrm{M}+\mathrm{HCOO}^{-}\right]^{-}} \\
{[\mathrm{M}-\mathrm{H}]^{-}}\end{array}$ & $\begin{array}{r}323.0769 \\
161.0243^{\mathrm{b}}\end{array}$ & $\mathrm{C}_{15} \mathrm{H}_{16} \mathrm{O}_{8}$ & 0.64 & 0.21 & Skimmin \# & Pharbitis nil & [34] \\
\hline 15 & 7.97 & n.d. & $\begin{array}{l}395.1930 \\
349.1880\end{array}$ & $\begin{array}{c}{\left[\mathrm{M}+\mathrm{HCOO}^{-}\right]^{-}} \\
{[\mathrm{M}-\mathrm{H}]^{-}}\end{array}$ & $\begin{array}{l}187.1341 ; \\
161.0457 ; \\
179.0563^{\mathrm{b}}\end{array}$ & $\mathrm{C}_{16} \mathrm{H}_{30} \mathrm{O}_{8}$ & 5.03 & 1.76 & $\begin{array}{l}\text { Monoterpenoid } \\
\text { glycoside I }\end{array}$ & & \\
\hline 16 & 8.66 & $\begin{array}{l}245 \\
324\end{array}$ & $\begin{array}{l}353.0881 \\
707.1868 \\
\end{array}$ & $\begin{array}{c}{[\mathrm{M}-\mathrm{H}]^{-}} \\
{[2 \mathrm{M}-\mathrm{H}]^{-}} \\
\end{array}$ & $\begin{array}{l}191.0560 \\
179.0350^{\mathrm{b}}\end{array}$ & $\mathrm{C}_{16} \mathrm{H}_{18} \mathrm{O}_{9}$ & 2.39 & 0.84 & $O$-caffeoylquinic acid ${ }^{\#}$ & $\begin{array}{l}\text { Ipomoea } \\
\text { batatas }\end{array}$ & [35] \\
\hline 17 & 9.06 & 288 & $\begin{array}{l}433.2091 \\
387.2014\end{array}$ & $\begin{array}{c}{\left[\mathrm{M}+\mathrm{HCOO}^{-}\right]^{-}} \\
{[\mathrm{M}-\mathrm{H}]^{-}}\end{array}$ & $161.0455^{b}$ & $\mathrm{C}_{19} \mathrm{H}_{32} \mathrm{O}_{8}$ & -2.88 & -1.12 & $\begin{array}{c}\text { Magastigmane } \\
\text { glycoside derivative } \\
\text { III }{ }^{\#}\end{array}$ & & \\
\hline 18 & 10.20 & 287 & 387.1870 & {$[\mathrm{M}-\mathrm{H}]^{-}$} & $\begin{array}{l}207.1022 ; \\
163.1128 \\
369.1544^{\mathrm{b}}\end{array}$ & $\mathrm{C}_{15} \mathrm{H}_{32} \mathrm{O}_{11}$ & 0.94 & 0.36 & n.i. & & \\
\hline 19 & 10.51 & n.d. & $\begin{array}{l}431.1928 \\
385.1853 \\
\end{array}$ & $\begin{array}{c}{\left[\mathrm{M}+\mathrm{HCOO}^{-}\right]^{-}} \\
{[\mathrm{M}-\mathrm{H}]^{-}} \\
\end{array}$ & $\begin{array}{r}223.1337 ; \\
205.1233 ; \\
153.0922 ; \\
161.0457^{\text {b }} \\
\end{array}$ & $\mathrm{C}_{19} \mathrm{H}_{30} \mathrm{O}_{8}$ & 1.97 & 0.76 & Roseoside $\#$ & $\begin{array}{l}\text { Ipomoea } \\
\text { purpurea }\end{array}$ & [36] \\
\hline 20 & 11.36 & 322 & 297.0986 & {$[\mathrm{M}-\mathrm{H}]^{-}$} & $\begin{array}{r}179.0352 \\
135.0453^{\mathrm{b}} \\
\end{array}$ & $\mathrm{C}_{14} \mathrm{H}_{18} \mathrm{O}_{7}$ & 3.95 & 1.17 & n.i. & & \\
\hline 21 & 12.13 & 265 & $\begin{array}{l}441.1969 \\
395.1899 \\
\end{array}$ & $\begin{array}{c}{\left[\mathrm{M}+\mathrm{HCOO}^{-}\right]^{-}} \\
{[\mathrm{M}-\mathrm{H}]^{-}}\end{array}$ & n.s. & $\mathrm{C}_{17} \mathrm{H}_{32} \mathrm{O}_{10}$ & -4.61 & -1.82 & n.i. & & \\
\hline 22 & 12.28 & n.d. & 281.1397 & {$[\mathrm{M}-\mathrm{H}]^{-}$} & $\begin{array}{l}237.1488 ; \\
171.1180 ; \\
123.0817 ; \\
201.1284^{\text {b }}\end{array}$ & $\mathrm{C}_{15} \mathrm{H}_{22} \mathrm{O}_{5}$ & 2.85 & 0.80 & n.i. & & \\
\hline 23 & 12.97 & 258 & \begin{tabular}{|c|}
583.2027 \\
537.1964 \\
1187.3107 \\
1613.8181 \\
\end{tabular} & $\begin{array}{c}{\left[\mathrm{M}+\mathrm{HCOO}^{-}\right]^{-}} \\
{[\mathrm{M}-\mathrm{H}]^{-}} \\
{[2 \mathrm{M}-\mathrm{H}]^{-}} \\
{[\mathrm{M}-\mathrm{H}]^{-}}\end{array}$ & n.s. & $\mathrm{C}_{26} \mathrm{H}_{34} \mathrm{O}_{12}$ & -1.49 & -0.80 & n.i. & & \\
\hline 24 & 13.26 & $\begin{array}{l}242 \\
298 \\
324\end{array}$ & 179.0353 & {$[\mathrm{M}-\mathrm{H}]^{-}$} & $135.0453^{b}$ & $\mathrm{C}_{9} \mathrm{H}_{8} \mathrm{O}_{4}$ & 4.84 & 0.87 & Caffeic acid ${ }^{\#}$ & $\begin{array}{c}\text { C. } \\
\text { trabutianus }\end{array}$ & [37] \\
\hline 25 & 14.25 & $\begin{array}{l}255 \\
331\end{array}$ & 741.1902 & {$[\mathrm{M}-\mathrm{H}]^{-}$} & $\begin{array}{l}\text { 300.0269; } \\
609.1447 ; \\
301.0347 \\
591.1343 \\
271.0242 \text { b }\end{array}$ & $\mathrm{C}_{32} \mathrm{H}_{38} \mathrm{O}_{20}$ & 3.21 & 2.38 & $\begin{array}{l}\text { Quercetin-O-pentosyl- } \\
\text { hexosyl-hexoside }^{\#}\end{array}$ & & \\
\hline 26 & 14.33 & $\begin{array}{l}294 \\
326\end{array}$ & 367.1024 & {$[\mathrm{M}-\mathrm{H}]^{-}$} & $\begin{array}{r}191.0559 ; \\
173.0455 ; \\
193.0499^{\mathrm{b}} \\
\end{array}$ & $\mathrm{C}_{17} \mathrm{H}_{20} \mathrm{O}_{9}$ & -1.38 & -0.51 & Feruloyl quinic acid \# & & \\
\hline 27 & 14.78 & n.d. & 225.1138 & {$[\mathrm{M}-\mathrm{H}]^{-}$} & $\begin{array}{l}181.1234 ; \\
165.0921 ; \\
147.0816 ; \\
135.0816^{\mathrm{b}}\end{array}$ & $\mathrm{C}_{12} \mathrm{H}_{18} \mathrm{O}_{4}$ & 4.96 & 1.12 & Tuberonic acid ${ }^{\#}$ & $\begin{array}{l}\text { Dichondra } \\
\text { repens }\end{array}$ & [38] \\
\hline
\end{tabular}


Table 1. Cont.

\begin{tabular}{|c|c|c|c|c|c|c|c|c|c|c|c|}
\hline Code & $\begin{array}{c}t_{R} \\
{[\mathrm{~min}]}\end{array}$ & $\lambda_{\max }$ & $m / z$ & MS Major Ion(s) & $\begin{array}{c}\text { MS/MS } \\
\text { Fragments } \\
{[\mathrm{m} / \mathrm{z}]}\end{array}$ & $\begin{array}{l}\text { Molecular } \\
\text { Formula }\end{array}$ & $\begin{array}{c}\Delta \\
\text { ppm }\end{array}$ & $\begin{array}{c}\Delta \\
\mathrm{mDa}\end{array}$ & Putative Identification & $\begin{array}{c}\text { Isolated } \\
\text { Previously }\end{array}$ & Ref. \\
\hline 28 & 16.09 & n.d. & 725.1935 & {$[\mathrm{M}-\mathrm{H}]^{-}$} & $\begin{array}{r}593.1494 ; \\
575.1391 ; \\
284.0317 \\
285.0394 \\
327.0500^{\text {b }}\end{array}$ & $\mathrm{C}_{32} \mathrm{H}_{38} \mathrm{O}_{19}$ & 0.82 & 0.60 & $\begin{array}{l}\text { Kaempferol-O-pentosyl- } \\
\text { hexosyl-hexoside }\end{array}$ & & \\
\hline 29 & 16.54 & $\begin{array}{l}254 \\
347\end{array}$ & $\begin{array}{c}609.1460 \\
1219.3008 \\
\end{array}$ & $\begin{array}{l}{[\mathrm{M}-\mathrm{H}]^{-}} \\
{[2 \mathrm{M}-\mathrm{H}]^{-}}\end{array}$ & $\begin{array}{r}301.0349 ; \\
300.0276 ; \\
271.0248 ; \\
343.0454^{\mathrm{b}} \\
\end{array}$ & $\mathrm{C}_{27} \mathrm{H}_{30} \mathrm{O}_{16}$ & 0.72 & 0.44 & Rutin * & $\begin{array}{l}\text { C. arvensis; } \\
\text { Cressa cretica }\end{array}$ & {$[15,39]$} \\
\hline 30 & 16.78 & n.d. & $\begin{array}{l}621.3154 \\
575.3090\end{array}$ & $\begin{array}{c}{\left[\mathrm{M}+\mathrm{HCOO}^{-}\right]^{-}} \\
{[\mathrm{M}-\mathrm{H}]^{-}}\end{array}$ & $557.2985^{\mathrm{b}}$ & $\mathrm{C}_{28} \mathrm{H}_{48} \mathrm{O}_{12}$ & 3.91 & 2.25 & n.i. & & \\
\hline 31 & 17.24 & n.d. & 665.3405 & {$\left[\mathrm{M}+\mathrm{HCOO}^{-}\right]^{-}$} & $\begin{array}{l}619.3352 ; \\
601.3245^{c}\end{array}$ & $\mathrm{C}_{31} \mathrm{H}_{54} \mathrm{O}_{15}$ & 3.61 & 2.23 & n.i. & & \\
\hline 32 & 17.84 & n.d. & 709.3664 & {$\left[\mathrm{M}+\mathrm{HCOO}^{-}\right]^{-}$} & $\begin{array}{l}645.3510 \\
663.3615^{c}\end{array}$ & $\mathrm{C}_{32} \mathrm{H}_{56} \mathrm{O}_{14}$ & 2.45 & 1.74 & n.i. & & \\
\hline 33 & 18.00 & $\begin{array}{l}240 \\
301 \\
325\end{array}$ & 593.1503 & {$[\mathrm{M}-\mathrm{H}]^{-}$} & $\begin{array}{r}285.0398 ; \\
284.0325 ; \\
327.0507 ; \\
255.0297^{b}\end{array}$ & $\mathrm{C}_{27} \mathrm{H}_{30} \mathrm{O}_{15}$ & -0.58 & -0.35 & $\begin{array}{l}\text { Kaempferol-O-hexosyl- } \\
\text { pentoside }\end{array}$ & & \\
\hline 34 & 18.36 & 309 & 753.3912 & {$\left[\mathrm{M}+\mathrm{HCOO}^{-}\right]^{-}$} & $\begin{array}{l}689.3768 \\
707.3875^{c}\end{array}$ & $\mathrm{C}_{34} \mathrm{H}_{60} \mathrm{O}_{15}$ & 0.43 & 0.32 & n.i. & & \\
\hline 35 & 18.73 & $\begin{array}{l}238 \\
265 \\
342\end{array}$ & $\begin{array}{r}593.1503 \\
1187.3107\end{array}$ & $\begin{array}{c}{[\mathrm{M}-\mathrm{H}]^{-}} \\
{[2 \mathrm{M}-\mathrm{H}]^{-}}\end{array}$ & $\begin{array}{l}285.0369 \\
327.0500^{\mathrm{b}}\end{array}$ & $\mathrm{C}_{27} \mathrm{H}_{30} \mathrm{O}_{15}$ & -0.58 & -0.35 & $\begin{array}{l}\text { Kaempferol-3-O- } \\
\text { rutinoside *\# }\end{array}$ & C. dorycnium & [40] \\
\hline 36 & 19.20 & $\begin{array}{l}297 \\
342\end{array}$ & 607.1292 & {$[\mathrm{M}-\mathrm{H}]^{-}$} & $\begin{array}{r}463.0877 ; \\
505.0982 ; \\
545.1293 \\
301.0352^{\mathrm{b}}\end{array}$ & $\mathrm{C}_{27} \mathrm{H}_{28} \mathrm{O}_{16}$ & -1.17 & -0.71 & $\begin{array}{l}\text { Quercetin-O-[-O- } \\
\text { (hydroxy-3- } \\
\text { methylglutaryl)- } \\
\text { hexoside] }^{\#}\end{array}$ & & \\
\hline 37 & 19.56 & $\begin{array}{l}301 \\
325\end{array}$ & 447.0950 & {$[\mathrm{M}-\mathrm{H}]^{-}$} & $\begin{array}{r}284.0324 ; \\
285.0399 ; \\
327.0507 ; \\
255.0297^{\text {b }}\end{array}$ & $\mathrm{C}_{21} \mathrm{H}_{20} \mathrm{O}_{11}$ & 5.06 & 2.26 & $\begin{array}{l}\text { Kaempferol-3-O- } \\
\text { glucoside *\# }\end{array}$ & $\begin{array}{c}\text { C. } \\
\text { trabutianus }\end{array}$ & [37] \\
\hline 38 & 19.69 & $\begin{array}{l}238 \\
325\end{array}$ & $\begin{array}{c}515.1215 \\
1031.2482\end{array}$ & $\begin{array}{l}{[\mathrm{M}-\mathrm{H}]^{-}} \\
{[2 \mathrm{M}-\mathrm{H}]^{-}}\end{array}$ & $\begin{array}{r}353.0858 ; \\
173.0454 ; \\
335.0765 ; \\
179.0347^{\mathrm{b}} \\
\end{array}$ & $\mathrm{C}_{25} \mathrm{H}_{24} \mathrm{O}_{12}$ & 4.95 & 2.55 & $\begin{array}{l}\text { 3,4-di-O-caffeoylquinic } \\
\text { acid }^{\#}\end{array}$ & $\begin{array}{c}\text { C. } \\
\text { trabutianus }\end{array}$ & [37] \\
\hline 39 & 20.06 & n.d. & $\begin{array}{l}511.2174 \\
929.4974\end{array}$ & $\begin{array}{c}{\left[\mathrm{M}+\mathrm{HCOO}^{-}\right]^{-}} \\
{\left[2 \mathrm{M}-\mathrm{H}^{-}\right.}\end{array}$ & $\begin{array}{l}161.0245 ; \\
179.0350^{c}\end{array}$ & $\mathrm{C}_{24} \mathrm{H}_{34} \mathrm{O}_{9}$ & -1.05 & -0.54 & n.i. & & \\
\hline 40 & 20.52 & n.d. & 973.5257 & {$[\mathrm{M}-\mathrm{H}]^{-}$} & $909.508^{b}$ & $\mathrm{C}_{46} \mathrm{H}_{78} \mathrm{~N}_{4} \mathrm{O}_{18}$ & 2.48 & 2.41 & n.i. & & \\
\hline 41 & 20.96 & $\begin{array}{l}245 ; \\
295 ; \\
327\end{array}$ & $\begin{array}{c}515.1211 \\
561.1236 \\
1031.2471 \\
\end{array}$ & $\begin{array}{c}{[\mathrm{M}-\mathrm{H}]^{-}} \\
{\left[\mathrm{M}+\mathrm{HCOO}^{-}\right]^{-}} \\
{[2 \mathrm{M}-\mathrm{H}]^{-}}\end{array}$ & $\begin{array}{c}353.0868 ; \\
191.056 ; \\
179.0349^{\mathrm{b}}\end{array}$ & $\mathrm{C}_{25} \mathrm{H}_{24} \mathrm{O}_{12}$ & 4.17 & 2.15 & $\begin{array}{l}\text { 3,5-di-O-caffeoylquinic } \\
\text { acid }^{* \#}\end{array}$ & $\begin{array}{c}\text { C. } \\
\text { trabutianus }\end{array}$ & [37] \\
\hline 42 & 21.67 & n.d. & 417.0831 & {$[\mathrm{M}-\mathrm{H}]^{-}$} & $\begin{array}{r}284.0323 ; \\
285.0397 \\
327.0506 ; \\
255.0295^{\text {b }}\end{array}$ & $\mathrm{C}_{20} \mathrm{H}_{18} \mathrm{O}_{10}$ & 2.23 & 1.80 & $\begin{array}{l}\text { Kaempferol-O- } \\
\text { pentoside }\end{array}$ & & \\
\hline 43 & 21.86 & $\begin{array}{l}245 \\
293 \\
324\end{array}$ & $\begin{array}{r}515.1214 \\
1031.2463\end{array}$ & $\begin{array}{c}{[\mathrm{M}-\mathrm{H}]^{-}} \\
{[2 \mathrm{M}-\mathrm{H}]^{-}}\end{array}$ & $\begin{array}{c}353.0866 ; \\
299.056 ; \\
173.0457 \\
203.0350 ; \\
191.0560^{\mathrm{b}}\end{array}$ & $\mathrm{C}_{25} \mathrm{H}_{24} \mathrm{O}_{12}$ & 2.37 & 0.93 & $\begin{array}{l}\text { 4,5-di-O-caffeoylquinic } \\
\text { acid }^{\#}\end{array}$ & & \\
\hline 44 & 22.49 & n.d. & 187.0981 & {$[\mathrm{M}-\mathrm{H}]^{-}$} & $\begin{array}{l}\text { 125.0975; } \\
97.0663^{\mathrm{b}}\end{array}$ & $\mathrm{C}_{9} \mathrm{H}_{16} \mathrm{O}_{4}$ & 5.70 & 1.07 & Azelaic acid ${ }^{\#}$ & & \\
\hline 45 & 22.93 & n.d. & $\begin{array}{l}625.2126 \\
579.2072\end{array}$ & $\begin{array}{c}{\left[\mathrm{M}+\mathrm{HCOO}^{-}\right]^{-}} \\
{[\mathrm{M}-\mathrm{H}]^{-}}\end{array}$ & n.s. & $\mathrm{C}_{28} \mathrm{H}_{36} \mathrm{O}_{13}$ & -0.98 & -0.57 & n.i. & & \\
\hline 46 & 24.91 & n.d. & 529.1365 & {$[\mathrm{M}-\mathrm{H}]^{-}$} & $\begin{array}{c}353.0868 ; \\
367.1023 ; \\
191.05611^{\mathrm{b}}\end{array}$ & $\mathrm{C}_{26} \mathrm{H}_{26} \mathrm{O}_{12}$ & 3.59 & 1.90 & $\begin{array}{l}\text { Caffeoyl-feruloyl quinic } \\
\text { acid isomer I }\end{array}$ & & \\
\hline 47 & 25.41 & n.d. & 529.1372 & {$[\mathrm{M}-\mathrm{H}]^{-}$} & $\begin{array}{l}367.1016 ; \\
173.0454 ; \\
193.0506 ; \\
179.0349^{\mathrm{b}}\end{array}$ & $\mathrm{C}_{26} \mathrm{H}_{26} \mathrm{O}_{12}$ & 4.91 & 2.60 & $\begin{array}{l}\text { Caffeoyl-feruloyl quinic } \\
\text { acid isomer II }\end{array}$ & & \\
\hline 48 & 26.09 & $\begin{array}{l}292 \\
305\end{array}$ & $\begin{array}{l}282.1146 \\
328.1194\end{array}$ & $\begin{array}{c}{[\mathrm{M}-\mathrm{H}]^{-}} \\
{\left[\mathrm{M}+\mathrm{HCOO}^{-}\right]^{-}}\end{array}$ & $\begin{array}{c}119.0506 ; \\
145.0296 \\
162.0563^{\mathrm{b}}\end{array}$ & $\mathrm{C}_{17} \mathrm{H}_{17} \mathrm{NO}_{3}$ & 5.61 & 1.58 & $\begin{array}{c}N \text {-trans }-p \text { - } \\
\text { coumaroyltyramine }\end{array}$ & $\begin{array}{l}\text { Ipomoea } \\
\text { batatas }\end{array}$ & [41] \\
\hline
\end{tabular}


Table 1. Cont.

\begin{tabular}{|c|c|c|c|c|c|c|c|c|c|c|c|}
\hline Code & $\begin{array}{c}t_{R} \\
{[\mathrm{~min}]}\end{array}$ & $\lambda_{\max }$ & $m / z$ & MS Major Ion(s) & $\begin{array}{c}\text { MS/MS } \\
\text { Fragments } \\
{[\mathrm{m} / \mathrm{z}]}\end{array}$ & $\begin{array}{l}\text { Molecular } \\
\text { Formula }\end{array}$ & $\begin{array}{c}\Delta \\
\text { ppm }\end{array}$ & $\underset{\mathrm{mDa}}{\Delta}$ & Putative Identification & $\begin{array}{c}\text { Isolated } \\
\text { Previously }\end{array}$ & Ref. \\
\hline 49 & 26.67 & $\begin{array}{l}238 ; \\
293 \\
318 \\
\end{array}$ & $\begin{array}{l}312.1243 \\
358.1294\end{array}$ & $\begin{array}{c}{[\mathrm{M}-\mathrm{H}]^{-}} \\
{\left[\mathrm{M}+\mathrm{HCOO}^{-}\right]^{-}}\end{array}$ & $\begin{array}{r}178.0512 ; \\
297.1007 ; \\
135.0454^{\mathrm{b}} \\
\end{array}$ & $\mathrm{C}_{18} \mathrm{H}_{19} \mathrm{NO}_{4}$ & 2.30 & 0.72 & $\begin{array}{c}N \text {-trans- } \\
\text { feruloyltyramine *\# }\end{array}$ & $\begin{array}{l}\text { Ipomoea } \\
\text { batatas }\end{array}$ & [41] \\
\hline 50 & 28.04 & n.d. & 607.2026 & {$\left[\mathrm{M}+\mathrm{HCOO}^{-}\right]^{-}$} & $561.1960^{c}$ & $\mathrm{C}_{28} \mathrm{H}_{34} \mathrm{O}_{12}$ & -0.13 & -0.08 & n.i. & & \\
\hline 51 & 29.36 & n.d. & 327.2182 & {$[\mathrm{M}-\mathrm{H}]^{-}$} & $\begin{array}{l}229.1438 \\
291.1964 \\
211.1336 ; \\
171.1029^{b}\end{array}$ & $\mathrm{C}_{18} \mathrm{H}_{32} \mathrm{O}_{5}$ & 3.21 & 1.05 & $\begin{array}{l}\text { Trihydroxy-10,15- } \\
\text { octadecadienoic acid } \\
\text { derivative I }{ }^{\#}\end{array}$ & & \\
\hline 52 & 30.56 & n.d. & 327.2178 & {$[\mathrm{M}-\mathrm{H}]^{-}$} & $\begin{array}{r}229.1437 \\
291.1961 ; \\
171.1027 \\
211.1336 \\
309.2067^{b}\end{array}$ & $\mathrm{C}_{18} \mathrm{H}_{32} \mathrm{O}_{5}$ & 1.99 & 0.65 & $\begin{array}{l}\text { Trihydroxy-10,15- } \\
\text { octadecadienoic acid } \\
\text { derivative II }\end{array}$ & & \\
\hline 53 & 31.36 & n.d. & 329.2329 & {$[\mathrm{M}-\mathrm{H}]^{-}$} & $\begin{array}{r}329.2333 \\
229.1440 \\
211.1337 \\
171.1030 \\
311.2228 \\
293.2122^{b}\end{array}$ & $\mathrm{C}_{18} \mathrm{H}_{34} \mathrm{O}_{5}$ & 0.31 & 0.10 & $\begin{array}{l}\text { Trihydroxy-10- } \\
\text { octadecenoic } \\
\text { acid }^{\#}\end{array}$ & & \\
\hline 54 & 31.90 & n.d. & $\begin{array}{l}883.4227 \\
837.4142\end{array}$ & $\begin{array}{c}{\left[\mathrm{M}+\mathrm{HCOO}^{-}\right]^{-}} \\
{[\mathrm{M}-\mathrm{H}]^{-}}\end{array}$ & $\begin{array}{l}561.6332 ; \\
533.2184^{\mathrm{b}}\end{array}$ & $\mathrm{C}_{39} \mathrm{H}_{66} \mathrm{O}_{19}$ & 2.62 & 2.20 & $\begin{array}{l}\text { O-(Hexosyl-hexosyl- } \\
\text { hexosyl)- } \\
\text { octadecatrienoyl- }^{\text {glycerol }}{ }^{\#}\end{array}$ & & \\
\hline 55 & 32.54 & n.d. & $\begin{array}{l}721.3635 \\
675.3586 \\
712.5355\end{array}$ & $\begin{array}{c}{\left[\mathrm{M}+\mathrm{HCOO}^{-}\right]^{-}} \\
{[\mathrm{M}-\mathrm{H}]^{-}} \\
{[\mathrm{M}-\mathrm{H}]^{-}}\end{array}$ & $397.1340^{\mathrm{b}}$ & $\mathrm{C}_{33} \mathrm{H}_{56} \mathrm{O}_{14}$ & -0.86 & -0.58 & $\begin{array}{l}O \text {-(Hexosyl-hexosyl)-O- } \\
\text { linolenoyl-glycerol }^{\#}\end{array}$ & & \\
\hline 56 & 33.05 & n.d. & $\begin{array}{l}647.3270 \\
601.3214\end{array}$ & $\begin{array}{c}{\left[\mathrm{M}+\mathrm{HCOO}^{-}\right]^{-}} \\
{[\mathrm{M}-\mathrm{H}]^{-}}\end{array}$ & n.s. & $\mathrm{C}_{30} \mathrm{H}_{50} \mathrm{O}_{12}$ & -1.67 & -1.00 & n.i. & & \\
\hline 57 & 33.53 & 218 & 559.3091 & {$\left[\mathrm{M}+\mathrm{HCOO}^{-}\right]^{-}$} & $\begin{array}{l}277.2170 \\
253.0926^{c}\end{array}$ & $\mathrm{C}_{27} \mathrm{H}_{46} \mathrm{O}_{9}$ & -4.89 & -2.74 & $\begin{array}{c}\text { O-Hexosyl-O- } \\
\text { linolenoyl-glycerol }\end{array}$ & & \\
\hline 58 & 34.56 & 215 & 699.3825 & {$\left[\mathrm{M}+\mathrm{HCOO}^{-}\right.$} & $397.1340^{c}$ & $\mathrm{C}_{31} \mathrm{H}_{58} \mathrm{O}_{14}$ & 3.13 & 2.19 & $\begin{array}{l}\text { O-(Hexosyl-hexosyl)-O- } \\
\text { palmitoyl-glycerol }^{\#}\end{array}$ & & \\
\hline 59 & 35.20 & n.d. & $\begin{array}{l}1659.8471 \\
1613.8181\end{array}$ & $\begin{array}{c}{\left[\mathrm{M}+\mathrm{HCOO}^{-}\right]^{-}} \\
{[\mathrm{M}-\mathrm{H}]^{-}}\end{array}$ & n.s. & $\mathrm{C}_{98} \mathrm{H}_{118} \mathrm{O}_{20}$ & 2.65 & 4.28 & n.i. & & \\
\hline 60 & 35.87 & 218 & $\begin{array}{l}819.5250 \\
773.5198\end{array}$ & $\begin{array}{c}{\left[\mathrm{M}+\mathrm{HCOO}^{-}\right]^{-}} \\
{[\mathrm{M}-\mathrm{H}]^{-}}\end{array}$ & $\begin{array}{l}773.5198 \\
277.2170 \\
513.3065^{b}\end{array}$ & $\mathrm{C}_{45} \mathrm{H}_{74} \mathrm{O}_{10}$ & -0.74 & -0.57 & $\begin{array}{c}O \text {-Hexosyl-di-O- } \\
\text { linolenoyl-glycerol }\end{array}$ & & \\
\hline 61 & 38.25 & 409 & $\begin{array}{l}997.5783 \\
951.5723\end{array}$ & $\begin{array}{c}{\left[\mathrm{M}+\mathrm{HCOO}^{-}\right]^{-}} \\
{[\mathrm{M}-\mathrm{H}]^{-}}\end{array}$ & n.s. & $\mathrm{C}_{51} \mathrm{H}_{84} \mathrm{O}_{16}$ & 4.40 & 4.19 & n.i. & & \\
\hline 62 & 38.70 & 218 & 765.5196 & {$[\mathrm{M}-\mathrm{H}]^{-}$} & $\begin{array}{l}505.3003 \\
255.2320 \\
277.2163 \\
527.2846 \\
747.5024\end{array}$ & $\mathrm{C}_{43} \mathrm{H}_{74} \mathrm{O}_{11}$ & 5.63 & 4.31 & n.i. & & \\
\hline 63 & 40.11 & 409 & 591.2607 & {$[\mathrm{M}-\mathrm{H}]^{-}$} & $\begin{array}{l}559.2329 \\
515.2441^{b}\end{array}$ & $\mathrm{C}_{34} \mathrm{H}_{40} \mathrm{O}_{9}$ & 2.19 & 1.29 & n.i. & & \\
\hline 64 & 40.69 & n.d. & 1835.8597 & {$[\mathrm{M}-\mathrm{H}]^{-}$} & n.s. & $\mathrm{C}_{116} \mathrm{H}_{124} \mathrm{O}_{20}$ & -0.58 & -1.07 & n.i. & & \\
\hline 65 & 41.44 & n.d. & $\begin{array}{l}758.5413 \\
712.5355\end{array}$ & $\begin{array}{c}{\left[\mathrm{M}+\mathrm{HCOO}^{-}\right]^{-}} \\
{[\mathrm{M}-\mathrm{H}]^{-}}\end{array}$ & n.s. & $\mathrm{C}_{41} \mathrm{H}_{71} \mathrm{~N}_{5} \mathrm{O}_{5}$ & -3.08 & -2.20 & n.i. & & \\
\hline
\end{tabular}

${ }^{a}$ Previously isolated from Convolvulaceae family species; ${ }^{\mathrm{b}}$ fragments produced from $[\mathrm{M}-\mathrm{H}]^{-}$adduct; ${ }^{c}$ fragments produced from $\left[\mathrm{M}+\mathrm{HCOO}^{-}\right]^{-}$adduct; n.d., not detectable by overlapping with near chromatographic compounds; n.s., no significant signals; n.i., no identified compound; * compounds identified by standard comparison. " compounds identified in C. arvensis for the first time.

The presence of compounds $13,29,35,37,41,48$, and 49 was further supported by co-injection with commercial standards. To the best of our knowledge, nineteen phenolic compounds $(4,10,14,16,19,25,26,28,33,35,36,38,41-43$, and 46-49) are hereby potentially characterized for the first time in C. arvensis by molecular networking. Resin glycosides and alkaloids were detected neither in ARE nor in ARM. 


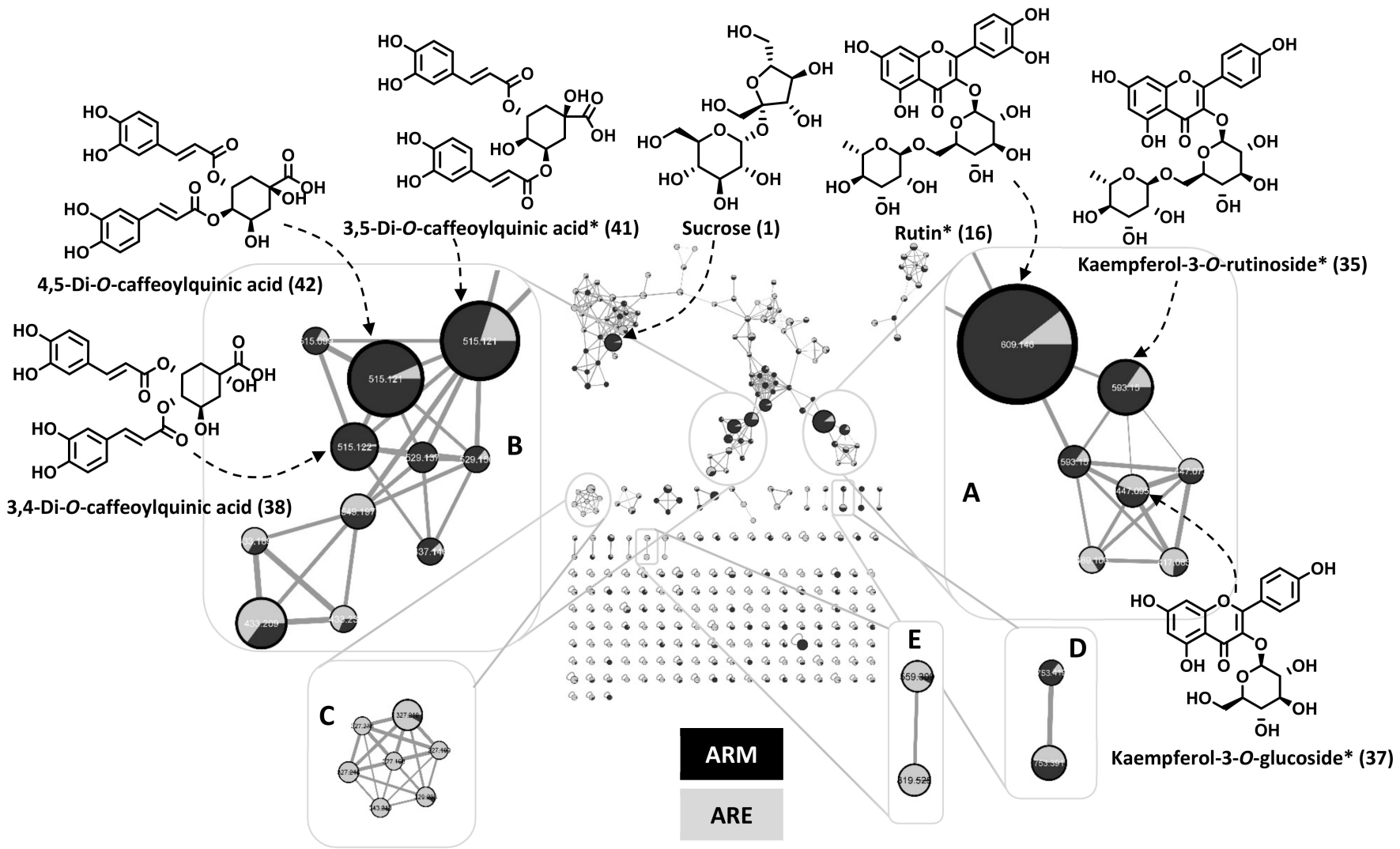

Figure 3. Molecular network of $C$. arvensis extracts (ARE and ARM). A: flavonoid glycosides; B: phenolic acids; C: lipid acids, D: flavonoid triglycosides; E: lipid glycosides. Clusters were built with a cosine of 0.7 with a minimum of 4 matched peaks. Size of nodes are proportional to corresponding peak area. Edge width are proportional to the corresponding cosine value. Compounds marked with * were additionally identified by standard comparison.

\subsection{Quantification and Biological Screening of Major Compounds of ARE and ARM}

To go one step further, the quantification analysis of main UV-absorbing constituents detected in both extracts was done by HPLC-PDA (Figure 4). Thus, chlorogenic acid (13), rutin (29), kaempferol-3-O-rutinoside (35), 3,5-di-O-caffeoylquinic acid (41), and $\mathrm{N}$ trans-p-coumaroyltyramine (48) were quantified using their respective authentic standards (Table 2). 3,4-di-O-caffeoylquinic acid (38), 4,5-di-O-caffeoylquinic acid (43), and $\mathrm{N}$-transferuloyltyramine (49) were quantified as equivalent of 3,5-di-O-caffeoylquinic acid (41) and $N$-trans-p-coumaroyltyramine (48), respectively, due to the lack of adequate standards quantities (Table 3). Chlorogenic acid (13), rutin (29), and 3,5-di-O-caffeoylquinic acid (41) were found as main constituents of ARM with the presence of $29.86 \pm 0.17,27.71 \pm 0.19$, and $38.16 \pm 0.13 \mathrm{mg} / \mathrm{g}$ of dry extract, respectively. For ARE, the tyramines derivatives $(48,49)$ and the 3,5-di-O-caffeoylquinic acid (41) were found as main constituents with amounts of $5.90 \pm 0.27,6.61 \pm 0.40$, and $5.82 \pm 0.32 \mathrm{mg} / \mathrm{g}$ of dry extract, respectively. Sugars and derivatives, as well as lipids and glycolipids, not absorbing in UV, are not quantifiable by this method. Based on these amounts, we calculated the concentration of these compounds in the assay we performed on the J774 cells where we evaluated the effect of ARE and ARM on the expression of pro-inflammatory mediators in LPS-stimulated J744 cells (Tables 2 and 3 ). 


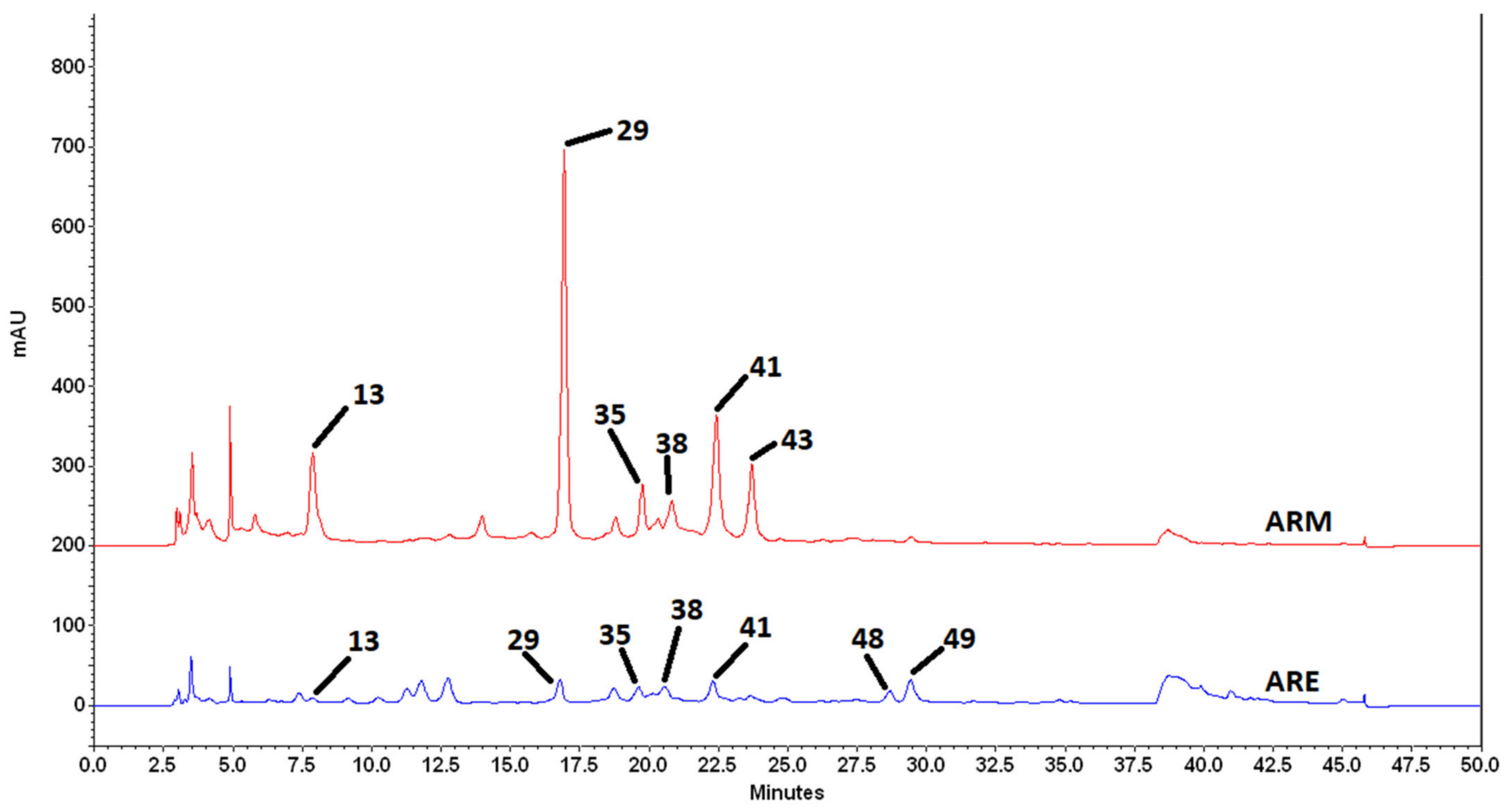

Figure 4. UV chromatogram of ARE and ARM at $254 \mathrm{~nm}$. Chlorogenic acid (13), rutin (29), kaempferol-3-O-rutinoside (35), 3,4-di-O-caffeoylquinic acid (38), 3,5-di-O-caffeoylquinic acid (41), 4,5di-O-caffeoylquinic acid (43), $N$-trans-p-coumaroyltyramine (48), and $N$-trans-feruloyltyramine (49).

Table 2. Major phenolic compounds quantified in ARE and ARM.

\begin{tabular}{|c|c|c|c|c|c|c|c|c|c|c|c|}
\hline \multirow[t]{2}{*}{ ID } & \multirow[t]{2}{*}{ Compound } & \multirow{2}{*}{$\begin{array}{c}\text { Regression } \\
\text { Equation }\end{array}$} & \multirow[t]{2}{*}{$\mathbf{R}^{2}$} & \multirow{2}{*}{$\begin{array}{l}\text { LOD } \\
(\mu \mathrm{g} / \mathrm{mL})\end{array}$} & \multirow{2}{*}{$\begin{array}{l}\text { LOQ } \\
(\mu \mathrm{g} / \mathrm{mL})\end{array}$} & \multirow{2}{*}{$\begin{array}{l}\text { Conc. in } \\
\text { Crude Extract } \\
\text { (mg/g Dry } \\
\text { Extract) }\end{array}$} & \multicolumn{2}{|c|}{$\begin{array}{l}\text { ARE } \\
\text { Concentration in } 50 \mu \mathrm{g} \\
\quad \text { of Extract } / \mathrm{mL}\end{array}$} & \multirow{2}{*}{$\begin{array}{c}\text { Conc. in } \\
\text { Crude Extract } \\
\text { (mg/g Dry } \\
\text { Extract) }\end{array}$} & \multicolumn{2}{|c|}{$\begin{array}{l}\text { ARM } \\
\text { Concentration in } 50 \mu \mathrm{g} \\
\quad \text { of Extract } / \mathrm{mL}\end{array}$} \\
\hline & & & & & & & $(\mu \mathrm{g} / \mathrm{mL})$ & $(\mu \mathrm{M})$ & & $(\mu \mathrm{g} / \mathrm{mL})$ & $(\mu \mathrm{M})$ \\
\hline 13 & Chlorogenic acid & $\begin{array}{c}y=40320 x+ \\
99146\end{array}$ & 0.9996 & 4.37 & 13.24 & $1.51 \pm 0.04$ & $\begin{array}{c}0.08 \pm \\
0.00\end{array}$ & $\begin{array}{c}0.21 \pm \\
0.01\end{array}$ & $29.86 \pm 0.17$ & $\begin{array}{c}1.49 \pm \\
0.01\end{array}$ & $\begin{array}{c}4.22 \pm \\
0.02\end{array}$ \\
\hline 29 & Rutin & $\begin{array}{c}y=26787 x+ \\
147669\end{array}$ & 0.9997 & 5.11 & 15.48 & $0.78 \pm 0.19$ & $\begin{array}{c}0.04 \pm \\
0.01\end{array}$ & $\begin{array}{c}0.06 \pm \\
0.02\end{array}$ & $27.71 \pm 0.19$ & $\begin{array}{c}1.39 \pm \\
0.01\end{array}$ & $\begin{array}{c}2.27 \pm \\
0.02\end{array}$ \\
\hline 35 & $\begin{array}{l}\text { Kaempferol-3-O- } \\
\text { rutinoside }\end{array}$ & $\begin{array}{c}y=36219 x+ \\
52756\end{array}$ & 0.9996 & 4.16 & 12.60 & $2.18 \pm 0.02$ & $\begin{array}{c}0.11 \pm \\
0.00\end{array}$ & $\begin{array}{c}0.18 \pm \\
0.00\end{array}$ & $5.23 \pm 0.19$ & $\begin{array}{c}0.26 \pm \\
0.01\end{array}$ & $\begin{array}{c}0.44 \pm \\
0.02\end{array}$ \\
\hline 41 & $\begin{array}{l}\text { 3,5-di-O- } \\
\text { caffeoylquinic } \\
\text { acid }\end{array}$ & $\begin{array}{c}y=59073 x+ \\
308772\end{array}$ & 0.9971 & 8.68 & 26.32 & $5.82 \pm 0.32$ & $\begin{array}{c}0.29 \pm \\
0.02\end{array}$ & $\begin{array}{c}0.56 \pm \\
0.03\end{array}$ & $38.16 \pm 0.13$ & $\begin{array}{c}1.91 \pm \\
0.01\end{array}$ & $\begin{array}{c}3.70 \pm \\
0.01\end{array}$ \\
\hline 48 & $\begin{array}{c}N \text {-trans- } p \text { - } \\
\text { coumaroyltyramine }\end{array}$ & $\begin{array}{c}y=88859 x+ \\
21246\end{array}$ & 0.9997 & 1.81 & 5.47 & $5.90 \pm 0.27$ & $\begin{array}{c}0.30 \pm \\
0.01\end{array}$ & $\begin{array}{c}1.04 \pm \\
0.05\end{array}$ & $1.72 \pm 0.13$ & $\begin{array}{c}0.09 \pm \\
0.01\end{array}$ & $\begin{array}{c}0.30 \pm \\
0.02\end{array}$ \\
\hline
\end{tabular}

Table 3. Relative content of major phenolic compounds of ARE and ARM, expressed as: a $\mathrm{mg}$ of 3,5-di-O-caffeoylquinic acid equivalent; ${ }^{b} \mathrm{mg}$ of $\mathrm{N}$-trans- $p$-coumaroyltyramine equivalent.

\begin{tabular}{|c|c|c|c|c|c|c|c|}
\hline ID & Compound & $\begin{array}{c}\text { Conc. in } \\
\text { Crude Extract } \\
\text { (mg/g Dry Extract) }\end{array}$ & \multicolumn{2}{|c|}{$\begin{array}{l}\text { ARE } \\
\text { Concentration in } 50 \mu \mathrm{g} \text { of Extract } / \mathrm{mL}\end{array}$} & $\begin{array}{c}\text { Conc. in } \\
\text { Crude Extract } \\
\text { (mg/g Dry Extract) }\end{array}$ & \multicolumn{2}{|c|}{$\begin{array}{l}\quad \text { ARM } \\
\text { Concentration in } 50 \mu \mathrm{g} \text { of Extract } / \mathrm{mL}\end{array}$} \\
\hline 38 & $\begin{array}{l}\text { 3,4-di-O-caffeoylquinic } \\
\text { acid }\end{array}$ & $1.48 \pm 0.04^{\mathrm{a}}$ & $0.07 \pm 0.00^{\mathrm{a}}$ & $0.14 \pm 0.00^{\mathrm{a}}$ & $14.08 \pm 0.81^{\mathrm{a}}$ & $0.704 \pm 0.04^{\mathrm{a}}$ & $1.36 \pm 0.08^{\mathrm{a}}$ \\
\hline 43 & $\begin{array}{l}\text { 4,5-di-O-caffeoylquinic } \\
\text { acid }\end{array}$ & $1.97 \pm 0.03^{\mathrm{a}}$ & $0.10 \pm 0.00^{a}$ & $0.19 \pm 0.00^{\mathrm{a}}$ & $21.34 \pm 0.06^{\mathrm{a}}$ & $1.067 \pm 0.00^{\mathrm{a}}$ & $2.07 \pm 0.01^{\mathrm{a}}$ \\
\hline 49 & $\begin{array}{c}N \text {-trans- } \\
\text { feruloyltyramine }\end{array}$ & $6.61 \pm 0.40^{b}$ & $0.33 \pm 0.02^{b}$ & $1.06 \pm 0.06^{\mathrm{b}}$ & $1.37 \pm 0.30^{b}$ & $0.068 \pm 0.02^{b}$ & $0.22 \pm 0.05^{b}$ \\
\hline
\end{tabular}


Next, to assess whether the main identified compounds of ARE and ARM could explain the activity of the extracts, rutin (29), kaempferol-3-O-rutinoside (35), chlorogenic acid (13), 3,5-di-O-caffeoylquinic acid (41), $N$-trans-p-coumaroyltyramine (48), and N-transferuloyltyramine (49), at $5 \mu \mathrm{M}$ concentration, were tested on LPS-activated J774 cells. Additionally, a mixture of these compounds (13, 29, 35, 41, 48, 49) with the same concentration as in $50 \mu \mathrm{g} / \mathrm{mL}$ ARM was also tested to evaluate if they have some additive or synergistic effect against IL-6 production and can explain the activity observed (Figure 5). In our hands, rutin (29), chlorogenic acid (13), N-trans-p-coumaroyltyramine (48), and $N$-trans-feruloyltyramine (49) were the most active, with IL-6 levels representing $71.6 \pm 4.8$, $56.8 \pm 8.9,48 \pm 6.6$, and $60.9 \pm 4.6 \%$, respectively, of those found in the presence of vehicle. In the mixture, IL-6 levels were $43.6 \pm 5.8 \%$ compared to vehicle.

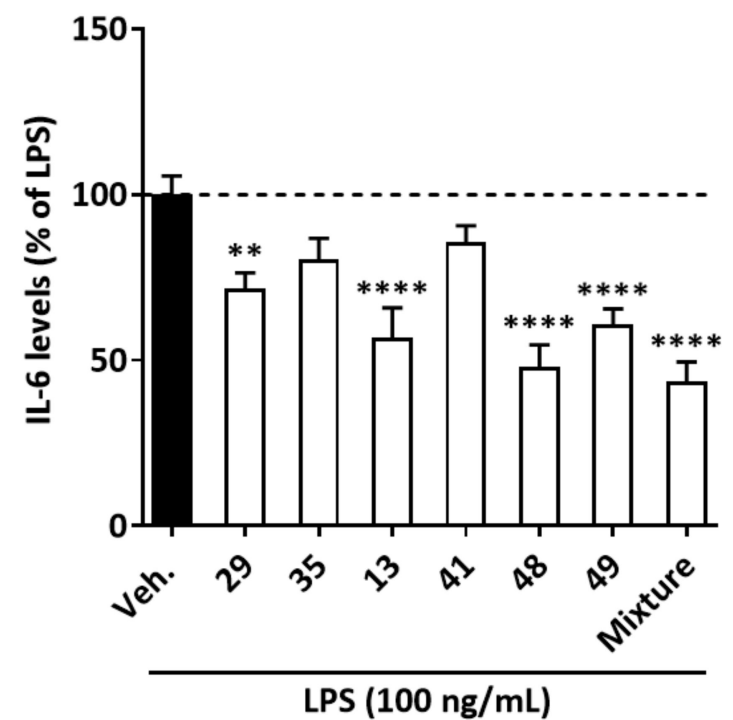

Figure 5. Effect of ARE, ARM, standards, and the mixture of standards on the expression of IL-6 in LPS-stimulated J744 cells. Cells were incubated with standards $(5 \mu \mathrm{M})$, their mixture (same concentration of standards as in $50 \mu \mathrm{g} / \mathrm{mL}$ ARM), or vehicle (Veh., $0.25 \%$ DMSO) and LPS (100 ng/mL) for $8 \mathrm{~h}$. IL-6 protein levels in the supernatant medium were quantified by ELISA. IL-6 was not detected in the medium of the unstimulated, vehicle-treated, cells. Results are expressed as percentage of the LPS-vehicle condition. The data were analyzed by the one-way ANOVA followed by Dunnet's post hoc test for comparisons between groups, are expressed as mean \pm SEM. $n=4$ in triplicates, ** $p<0.05,{ }^{* * *} p<0.0001$ vs. Veh. Rutin (29); kaempferol-3-O-rutinoside (35); chlorogenic acid (3-O-caffeoylquinic acid) (13); 3,5-di- $O$-caffeoylquinic acid (41); $N$-trans- $p$-coumaroyltyramine (48); $\mathrm{N}$-trans-feruloyltyramine (49); mixture $(29,35,13,41,48,49)$.

This is the first reported effect of $N$-trans- $p$-coumaroyltyramine (48) on pro-inflammatory cytokine production. Rutin (29) is an important dietary flavonoid with several therapeutic effects [42]. It was found to dose dependently $(25,50$, and $100 \mu \mathrm{M})$ suppress the activation of NF- $\kappa$ B and production of TNF- $\alpha$ in LPS-activated primary human umbilical vein endothelial cells (HUVECs) [43]. Chlorogenic acid (13) is an important biologically active phenolic compound with several therapeutic activities [44]. At $20 \mu \mathrm{M}$ concentration, it was found to decrease iNOS-mediated nitric oxide production, and to decrease pro-inflammatory cytokines (e.g., IL-1 $\beta$, TNF- $\alpha$, IL-6, and CXCL1) expression through down-regulation of NF-кB in LPS-stimulated RAW 264.7 macrophages [45]. Results obtained in our study show a significant effect of chlorogenic acid (13) on IL-6 production at $5 \mu \mathrm{M}$. N-transferuloyltyramine (49) (tested at $160 \mu \mathrm{M}$ ) was found to significantly decrease NO, PGE 2 , and ROS production and down-regulate iNOS and COX-2 mRNA expression in LPS-stimulated RAW 264.7 cells. These effects were associated with inactivation of AP-1 and MAPKs, which resulted from blocking of JNK phosphorylation [46]. Our data show a significant effect 
of rutin (29), chlorogenic acid (13), and N-trans-feruloyltyramine (49) on LPS-induced IL-6 production at $5 \mu \mathrm{M}$.

Moreover, the levels of IL- 6 production (as \% of LPS-vehicle condition) in ARM-treated and the mixture-treated cells are $27.3 \pm 3.8 \%$ (data taken from Figure $2 \mathrm{~F}$ ) and $43.6 \pm 5.8 \%$ (data taken from Figure 5), respectively. The stronger inhibitory effect of ARM on IL-6 production compared to the mixture suggests that, besides the most abundant ones, other compounds present in the ARM extract also contribute to the activity.

Chlorogenic acid (13), rutin (29), and tyramine derivatives $(48,49)$ are water-soluble compounds, but the decoction (ARW) did not inhibit the expression of IL-6 in our LPSactivated J774 cells model. In our HPLC-PDA analysis of ARW, we did not detect tyramine derivatives $(48,49)$, which may be degraded during boiling in water. Chlorogenic acid (13) and rutin (29) were detected and quantified as $28.23 \pm 0.86$ and $13.87 \pm 0.07 \mathrm{mg} / \mathrm{g}$ of dry extract, respectively. Two hypotheses could explain the absence of activity of ARW despite the presence of chlorogenic acid (13) and rutin (29). Firstly, 13, 29, 48, and 49 are not the only active compounds of ARE and ARM, rather there are also other compounds contributing to the anti-inflammatory activity of ARE and ARM. Secondly, ARW may contain some compounds which are antagonizing the effect of active compounds.

Finally, from a mechanistic perspective, as the crude extracts contain different chemical constituents and act by several synergistic mechanisms, further studies are required to study the effect of ARE and ARM on different inflammatory pathways. These could include NF$\mathrm{KB}$ and MAPK pathways as chlorogenic acid (13), rutin (29), and $N$-trans-feruloyltyramine (49) have already been reported to inhibit these pathways in LPS-activated macrophage in vitro models.

\section{Materials and Methods}

\subsection{Chemicals and Reagents}

HPLC grade acetonitrile, ethyl acetate, dichloromethane, hexane, and methanol were purchased from VWR International (Radnor, PA, USA). Kaempferol-3-O-rutinoside (35) and 3,5-di-O-caffeoylquinic acid (41) were purchased from Extrasynthèse (Genay, France). $N$-trans-p-coumaroyltyramine (48) was purchased from Phytolab GmbH \& Co. KG (Vestenbergsgreuth, Germany). Chlorogenic acid (3-O-caffeoylquinic acid) (13), rutin (29), Ntrans-feruloyltyramine (moupinamide) (49), dimethylsulfoxide (DMSO), camptothecin, (3-(4,5-dimethylthiazol-2-yl)-2,5-diphenyltetrazolium bromide (MTT), fetal bovine serum (FBS), and lipopolysaccharides (LPS from E. coli serotype O55:B5) were purchased from Sigma-Aldrich (Bornem, Belgium).

\subsection{Collection of Plant}

C. arvensis whole plant (aerial parts, roots, and flowers) was collected from the crop fields of Mouza As-haba, Jhang, Province Punjab, Pakistan in the month of April-May 2018. The collected plant material was authenticated by Dr. Zafarullah Zafar, Institute of Pure and Applied Biology/Botany Division, Bahauddin Zakariya University Multan, Pakistan. A voucher number (R. R. Stewart, F. W. Pak. 572(2) at BZU Pakistan and PAK-ZAFAR 002 at GNOS UCLouvain) was assigned for future reference. The plant name was further confirmed by checking in The Plant List (http:/ /www.theplantlist.org, accessed on 26 October 2019).

\subsection{Preparation of Crude Extracts}

The collected $C$. arvensis whole plants were washed with tap water, shade dried and then ground. Then, $50 \mathrm{~g}$ of the powdered plant material was extracted in a Soxhlet apparatus for $8 \mathrm{~h}$ by using successively $250 \mathrm{~mL}$ of hexane, dichloromethane, ethyl acetate, and methanol. The solvents were removed by rotary evaporator and four corresponding crude extracts (ARH, ARD, ARE, and ARM, respectively) were obtained. A decoction (ARW) was also prepared by boiling $50 \mathrm{~g}$ of the plant material in $1 \mathrm{~L}$ of water for $15 \mathrm{~min}$, 
filtered hot, and water was removed by lyophilization. The extracts and the decoction were stored at $-20{ }^{\circ} \mathrm{C}$ until further use.

\subsection{Cell Cultures}

Murine macrophage cell line J774 (a kind gift from Prof Van Bambeke, LDRI, UCLouvain) and human lung fibroblast cell line WI38 (ATCC CCL-75, bought from LGC standards, Molsheim, France) were grown in Roswell Park Memorial Institute medium (RPMI 1640 medium with GlutaMAX, Gibco, Thermo Fisher Scientific ${ }^{\circledR}$, Bleiswijk, The Netherlands) and Dulbecco's modified eagle medium (DMEM, containing $1 \mathrm{~g} / \mathrm{L}$ glucose and $1 \mathrm{mM}$ pyruvate, Gibco), respectively, supplemented with $10 \%$ fetal bovine serum (FBS) and penicillin-streptomycin (100 UI/mL) (Lonza, Verviers, Belgium), maintained at $37^{\circ} \mathrm{C}$ in $5 \%$ $\mathrm{CO}_{2}$ incubator.

\subsection{MTT Assay}

The crude extracts were analyzed by MTT assay on J774 and WI38 cells. Cells were seeded overnight in a 96-well plate at a density of $5 \times 10^{3}$ cells per well in $180 \mu \mathrm{L}$ per well of their respective culture medium. After $24 \mathrm{~h}$, cells were treated with stock solutions of crude extracts, diluted in the respective culture medium, $20 \mu \mathrm{L}$ per well, with the final concentration of $100 \mu \mathrm{g} / \mathrm{mL}$ and $50 \mu \mathrm{g} / \mathrm{mL}$, and incubated for further $72 \mathrm{~h}$. Based on their solubility, stock solutions of ARH, ARD, ARE, and ARM were prepared in DMSO while those of ARW in EtOH- $\mathrm{H}_{2} \mathrm{O}$ (25:75). At the end of the incubation, medium was removed and the cells were incubated for $45 \mathrm{~min}$ with $100 \mu \mathrm{L}$ of MTT solution prepared by dissolving $15 \mathrm{mg}$ of MTT in $5 \mathrm{~mL}$ of PBS and $45 \mathrm{~mL}$ of the respective culture medium. Next, MTT solution was replaced by DMSO (100 $\mu \mathrm{L}$ per well) and the absorbance was measured with a spectrophotometer (SpectraMax-Molecular Devices, Berkshire, UK) at $570 \mathrm{~nm}$ (with $620 \mathrm{~nm}$ reference wavelength) [47]. Camptothecin was used as a positive control while medium with vehicles (DMSO and EtOH- $\mathrm{H}_{2} \mathrm{O}(25: 75)$ ) at $0.5 \%$ were used as negative controls. All experiments were performed at least two times in triplicate.

\subsection{Effect of Crude Extracts and Identified Major Compounds on the Expression of Pro-Inflammatory Mediators in LPS-Stimulated J774 Cells}

J774 cells were seeded overnight in $1 \mathrm{~mL}$ per well of RPMI medium at the density of $2.5 \times 10^{5}$ cells/well in a 24-well plate. Then, the medium was removed and the cells were treated with the crude extracts solutions $(50 \mu \mathrm{g} / \mathrm{mL})$, or vehicle $(0.25 \%$ DMSO) with or without LPS $(100 \mathrm{ng} / \mathrm{mL})$ [48]. Identified main compounds of the extracts were tested at $5 \mu \mathrm{M}$ concentration and a mixture of them at the same concentration as in $50 \mu \mathrm{g} / \mathrm{mL}$ ARM. After $8 \mathrm{~h}$ incubation, supernatants were collected and stored at $-20^{\circ} \mathrm{C}$ for ELISA and cell culture plates were stored at $-80^{\circ} \mathrm{C}$.

\subsection{Real-Time Quantitative PCR ( $q P C R$ )}

Total RNA from the cells was extracted using TriPure reagent (Roche, Basel, Switzerland) according to the manufacturer's instructions. cDNA was synthesized from $1 \mu \mathrm{g}$ of total RNA using a reverse transcription kit (RT GoScript kit, Promega Benelux BV, Leiden, The Netherlands). Real-time qPCR analysis was performed on a QuantStudio 3 instrument (Applied Biosystems, Thermo Fisher Scientific ${ }^{\circledR}$, Bleiswijk, The Netherlands) using a SYBR Green mix (GoTaq qPCR Master mix, Promega). The following conditions were used for amplification: an initial holding stage of $10 \mathrm{~min}$ at $95^{\circ} \mathrm{C}$, then 45 cycles consisting of denaturation at $95^{\circ} \mathrm{C}$ for $3 \mathrm{~s}$, annealing at $60^{\circ} \mathrm{C}$ for $26 \mathrm{~s}$, and extension at $72{ }^{\circ} \mathrm{C}$ for $10 \mathrm{~s}$. At the end of the PCR reaction, melting curves of the products were obtained. The resulting cycle threshold $(\mathrm{Ct})$ were recorded for each gene and normalized using $60 \mathrm{~S}$ ribosomal protein L19 (RPL19) mRNA as reference. Results are expressed relative to control, using the "delta-delta Ct" method. Primer sequences are given in Table 4 [49]. 
Table 4. Primer sequences for qPCR.

\begin{tabular}{|c|c|c|}
\hline Gene/Product & Forward Primer $\left(5^{\prime}\right.$ to $\left.3^{\prime}\right)$ & Reverse Primer $\left(5^{\prime}\right.$ to $\left.3^{\prime}\right)$ \\
\hline Il6/IL-6 & ACAAGTCGGAGGCTTAATTACACAT & TTGCCATTGCACAACTCTTTTC \\
\hline $\operatorname{Tnf} / \mathrm{TNF}-\alpha$ & CTACTGAACTTCGGGGTGATC & TGAGTGTGAGGGTCTGGGC \\
\hline Ccl2/MCP-1 & GTCCCAAAGAAGCTGTAGTTTTTG & ATGTATGTCTGGACCCATTCC \\
\hline Rpl19/RPL19 & TGACCTGGATGAGAAGGATGAG & CTGTGATACATATGGCGGTCAATC \\
\hline Ptgs2/COX-2 & TGACCCCCAAGGCTCAAATAT & TGAACCCAGGTCCTCGCTTA \\
\hline Nos2/iNOS & AGGTACTCAGCGTGCTCCAC & GCACCGAAGATATCTTCATG \\
\hline
\end{tabular}

\subsection{Cytokines Quantification by ELISA}

Concentrations of IL- 6 , TNF- $\alpha$, and MCP- 1 in the collected cell culture supernatants were determined by a sandwich type ELISA technique using the Ready-Set-Go! Kit following the manufacturer's instructions (Invitrogen, Thermo Fisher Scientific ${ }^{\circledR}$, Bleiswijk, The Netherlands) [50].

\subsection{HPLC-PDA Analysis}

Chromatographic separation was performed on an HPLC-PDA system consisting of a Thermo Accela pump and PDA ray detector (Thermo Scientific ${ }^{\mathrm{TM}}$, Bremen, Germany). The LC separation was done on a Phenomenex Luna C18, $250 \times 4.6 \mathrm{~mm}$ packed with $5 \mu \mathrm{m}$ particles. Stock solutions of standards were prepared at $1 \mathrm{mg} / \mathrm{mL}$ concentration in HPLC grade methanol and then serially diluted to achieve five different concentrations in the range of: $500-5 \mu \mathrm{g} / \mathrm{mL}$ for $29 ; 200-5 \mu \mathrm{g} / \mathrm{mL}$ for $41 ; 50-1$ for 48 ; and $100-1 \mu \mathrm{g} / \mathrm{mL}$ for 13 and 35. All analyses were carried out with $20 \mu \mathrm{L}$ injection volume and with a flow rate of $0.8 \mathrm{~mL} / \mathrm{min}$. Detection wavelength was set between 200 to $400 \mathrm{~nm}$. The mobile phase consisted of $0.1 \%$ of formic acid in water (solvent A) and $100 \%$ acetonitrile (solvent B). Elution of the mobile phase was performed in gradient mode: $0-5 \min (17 \% \mathrm{~B})$; 5-35 min (17-40\% B); 35-36 min (40-100\% B); 36-42 $\min (100 \% \mathrm{~B}) ; 42-43 \mathrm{~min}(100-17 \% \mathrm{~B})$; and 43-50 min (17\% B). Chromatograms were integrated at $355 \mathrm{~nm}$ for 29 and 35; $290 \mathrm{~nm}$ for 48; and at $327 \mathrm{~nm}$ for 13 and 41. ARE and ARM solutions were freshly prepared at $8 \mathrm{mg} / \mathrm{mL}$ in HPLC grade methanol and analyzed under the same conditions as standards. LOD and LOQ were calculated from the residual standard deviation $(\sigma)$ of the regression curves and the slopes (S), according to the following equations: $\mathrm{LOD}=3.3 \mathrm{\sigma} / \mathrm{S}$ and $\mathrm{LOQ}=10 \mathrm{\sigma} / \mathrm{S}$.

\subsection{HPLC-DAD-HRMS/MS Analysis}

HPLC separations were conducted as described previously in HPLC-PDA analysis, and connected with a Thermo Scientific LTQ orbitrap XL mass spectrometer (Thermo Scientific $^{\mathrm{TM}}$, Bremen, Germany). The instrument was controlled using Thermo Scientific Xcalibur X software. The LC separation was done on a Phenomenex Luna C18, $250 \times 4.6 \mathrm{~mm}$ packed with $5 \mu \mathrm{m}$ particles. All analyses were carried out with $20 \mu \mathrm{L}$ injection volume, with the flow rate of $0.8 \mathrm{~mL} / \mathrm{min}$, and with the mobile phase consisting of $0.1 \%$ of formic acid in water (solvent A) and 100\% acetonitrile (solvent B). Elution of the mobile phase was performed in gradient mode: 0-5 min (17\% B); $5-27 \mathrm{~min}(17-40 \% \mathrm{~B})$; $27-28 \mathrm{~min}(40-100 \% \mathrm{~B})$; $28-38 \mathrm{~min}(100 \% \mathrm{~B}) ; 38-39 \mathrm{~min}(100-17 \% \mathrm{~B})$; and $39-50 \mathrm{~min}(17 \% \mathrm{~B})$. Chromatograms were recorded between 200 and $600 \mathrm{~nm}$. HRMS analyses were realized in APCI negative and positive modes with the following inlet conditions: for negative mode: capillary temperature $250{ }^{\circ} \mathrm{C}$; APCI vaporizer temperature $400{ }^{\circ} \mathrm{C}$; sheath gas flow 25 a.u.; auxiliary gas flow 25 a.u. and sweep gas flow 5 a.u. Discharge current of $5 \mu \mathrm{A}$; capillary temperature of $250^{\circ} \mathrm{C}$; capillary voltage of $-10 \mathrm{~V}$ and tube lens voltage of $-125 \mathrm{~V}$. For positive mode: capillary temperature $250{ }^{\circ} \mathrm{C}$; APCI vaporizer temperature $400{ }^{\circ} \mathrm{C}$; sheath gas flow 25 a.u.; auxiliary gas flow 25 a.u. and sweep gas flow 5 a.u.; discharge current of $5 \mu \mathrm{A}$; capillary temperature of $250{ }^{\circ} \mathrm{C}$; capillary voltage of $21 \mathrm{~V}$ and tube lens voltage of 
$75 \mathrm{~V}$. The data-dependent MS/MS events were performed on the three most intense ions detected in full scans MS.

\subsection{MS Data Treatment}

All HRMS run data (.RAW) files were exported to the open source software package MZmine 2 (2.53 version) for data processing [51]. For mass detection at $\mathrm{MS}^{1}$ level, the noise level was set to $1.0 \times 10^{5}$ for negative mode (APCI source) (negative mode was found to be more informative than positive mode). For $\mathrm{MS}^{2}$ detection, the noise level was set to 0 . The ADAP chromatogram builder was used and set to a minimum group size of scans of 4 , a minimum group intensity of $1.0 \times 10^{4}$, a minimum highest intensity of $1.0 \times 10^{5}$, and $m / z$ tolerance of $5 \mathrm{ppm}$. The ADAP algorithm (wavelets) was used for chromatogram deconvolution. The intensity window $\mathrm{S} / \mathrm{N}$ was used as an $\mathrm{S} / \mathrm{N}$ estimator with a signal to noise ratio set at 8 , a minimum feature height of $1.0 \times 10^{5}$, a coefficient area threshold at 10, a peak duration ranging from 0.02 to $0.8 \mathrm{~min}$, and the RT wavelet range from 0.02 to $0.2 \mathrm{~min}$. Isotopes were detected using the isotope peak grouper with a $\mathrm{m} / \mathrm{z}$ tolerance of $5 \mathrm{ppm}$, a RT tolerance of $0.02 \mathrm{~min}$ (absolute), the maximum charge set at 1 , and the representative isotope used was the most intense. Then, the aligned list peak was gap-filled with RT range of $0.04 \mathrm{~min}$ and $\mathrm{m} / z$ tolerance of $8 \mathrm{ppm}$. The resulting list was filtered using the peak list rows filter option to remove all the duplicates and all the features without $\mathrm{MS}^{2}$ spectrum associated.

\subsection{Mass Spectral Organization and Dereplication}

A molecular network was constructed from the .mgf file exported from MZmine, using the online workflow on the GNPS website [22]. The precursor ion mass tolerance was set to $0.02 \mathrm{Da}$ with a MS/MS fragment ion tolerance of $0.02 \mathrm{Da}$. A network was then created where edges were filtered to have a cosine of 0.7 and more than four matched peaks. The spectra in the network were then searched against GNPS's spectral libraries filtered under the same conditions as before. All matches kept between network spectra and library spectra were required to have a score of 0.75 and at least three matched peaks. Additional putative identification of unmatched peaks was carried out comparing available MS/MS fragmentation patterns in the literature. Data visualization was achieved using Cytoscape 3.8.0 [52]. Peak area data from the .csv file obtained from MZmine was added to the network. Size nodes were set proportionally to the total area of each peak detected in both analyzed extracts. Edge widths were set corresponding to the cosine score.

\section{Conclusions}

We report that ethyl acetate (ARE) and methanol (ARM) extracts of $C$. arvensis significantly inhibited the expression of pro-inflammatory mediators by activated J774 cells. Using a dereplication strategy, 45 compounds were putatively identified, among which rutin, kaempferol-3-O-rutinoside, chlorogenic acid (3-O-caffeoylquinic acid), 3,5-di-Ocaffeoylquinic acid, $\mathrm{N}$-trans-p-coumaroyltyramine, and $\mathrm{N}$-trans-feruloyltyramine were among the major compounds present in both active extracts. These compounds were quantified and tested on LPS-activated J774 cells where they were shown to be responsible for a part of the observed effects against IL-6 production. Taken together, our studies will contribute to a better understanding of the chemical composition and the biological properties of Convolvulus arvensis.

Author Contributions: Conceptualization, H.A.K., S.O., M.A., G.G.M. and J.Q.-L.; methodology, H.A.K., S.O. and M.A.; data curation, H.A.K., S.O. and M.A.; writing-original draft preparation, H.A.K.; writing-review and editing, H.A.K., S.O., M.A., G.G.M. and J.Q.-L.; supervision, G.G.M. and J.Q.-L.; project administration, G.G.M. and J.Q.-L. All authors have read and agreed to the published version of the manuscript. 
Funding: This research was funded by Higher Education Commission (HEC), Pakistan under the project "Faculty Development Program of Bahauddin Zakariya University Multan (100 PhD foreign scholarships) (Prime Minister's directive)".

Institutional Review Board Statement: Not applicable.

Informed Consent Statement: Not applicable.

Data Availability Statement: All data are available in the manuscript.

Acknowledgments: The authors gratefully acknowledge Higher Education Commission, Pakistan, for financial support under the project "Faculty Development Program of Bahauddin Zakariya University Multan (100 PhD foreign scholarships) (Prime Minister's directive)". The authors are grateful to Nasira Parveen for help in plant collection and to Zafarullah Zafar (Bahauddin Zakariya University Multan, Pakistan) for authentication of the plant. The authors wish to thank Hafsa Ameraoui for help in revision experiments, Marie-France Hérent and Owein Guillemot-Legris for skillful technical assistance and to all the members of the GNOS and BPBL for helpful scientific advice and support. MS data were obtained at the MASSMET platform of UCLouvain. M.A. is a postdoctoral researcher from the FRS-FNRS (Fonds de la Recherche Scientifique) Belgium.

Conflicts of Interest: The authors declare that they have no conflict of interest.

Sample Availability: Samples of the compounds are not available from the authors.

\section{References}

1. Uttra, A.M.; Ahsan, H.; Hasan, U.H.; Chaudhary, M.A. Traditional Medicines of Plant Origin Used for the Treatment of Inflammatory Disorders in Pakistan: A Review. J. Tradit. Chin. Med. 2018, 38, 636-656. [CrossRef]

2. Kaur, M.; Kalia, A.N. Convolvulus Arvensis-A Useful Weed. Int. J. Pharm. Pharm. Sci. 2012, 4, 38-40.

3. Iqbal, H.; Sher, Z.; Khan, Z.U. Medicinal Plants from Salt Range Pind Dadan Khan, District Jhelum, Punjab, Pakistan. J. Med. Plant. Res. 2011, 5, 2157-2168.

4. Ijaz, F.; Iqbal, Z.; Alam, J.; Khan, S.M.; Afzal, A.; Rahman, I.U.; Afzal, M.; Islam, M. Ethno Medicinal Study upon Folk Recipes against Various Human Diseases in Sarban Hills, Abbottabad, Pakistan. WJZ 2015, 10, 41-46. [CrossRef]

5. Ahmed, N.; Mahmood, A.; Mahmood, A.; Tahir, S.S.; Bano, A.; Malik, R.N.; Hassan, S.; Ishtiaq, M. Relative Importance of Indigenous Medicinal Plants from Layyah District, Punjab Province, Pakistan. J. Ethnopharmacol. 2014, 155, 509-523. [CrossRef] [PubMed]

6. Aslam, M.S.; Roy, S.D.; Ghaffari, M.A.; Uzair, M.; Ijaz, A.S.; Khan, T.R. Survey of Ethno-Medicinal Weeds of District Rajhan Pur, Punjab, Pakistan. Ind. Res. J. Pharm. Sci. 2014, 1, 38-45.

7. Abbas, Z.; Khan, S.M.; Abbasi, A.M.; Pieroni, A.; Ullah, Z.; Iqbal, M.; Ahmad, Z. Ethnobotany of the Balti Community, Tormik Valley, Karakorum Range, Baltistan, Pakistan. J. Ethnobiol. Ethnomed. 2016, 12, 1-15. [CrossRef]

8. Elzaawely, A.A.; Tawata, S. Antioxidant Activity of Phenolic Rich Fraction Obtained from Convolvulus arvensis L. Leaves Grown in Egypt. Asian J. Crop. Sci. 2012, 4, 32-40. [CrossRef]

9. Khakimov, Z.; Rakhmanov, A.K.; Yakubova, U.B.; Shukurlaev, K.S. Experimental Substantiation of Anti-Inflammatory Activity of a Gel Containing Convolvulus arvensis Extract in Carrageenan-Induced Aseptic Arthritis. Natl. J. Physiol. Pharm. Pharmacol. 2021, 11, 645-648. [CrossRef]

10. Saleem, U.; Zaib, S.; Khalid, S.; Anwar, F.; Akhtar, M.F.; Ahmad, B. Chemical Characterization, Docking Studies, Anti-Arthritic Activity and Acute Oral Toxicity of Convolvulus arvensis L. Leaves. Asian Pac. J. Trop. Biomed. 2020, 10, 442-451. [CrossRef]

11. Ali, M.; Qadir, M.I.; Saleem, M.; Janbaz, K.H.; Gul, H.; Hussain, L.; Ahmad, B. Hepatoprotective Potential of Convolvulus arvensis against Paracetamol-Induced Hepatotoxicity. Bangladesh J. Pharmacol. 2013, 8, 300-304. [CrossRef]

12. Awaad, A.S.; Mohamed, N.H.; El-Sayed, N.H. Phenolics of Convolvulus arvensis L. and Their Related Pharmacological Activity. Asian J. Chem. 2006, 18, 2818-2826.

13. Todd, F. Tropane Alkaloids and Toxicity of Convolvulus arvensis. Phytochemistry 1995, 39, 301-303. [CrossRef]

14. Fan, B.-Y.; He, Y.; Lu, Y.; Yang, M.; Zhu, Q.; Chen, G.-T.; Li, J.-L. Glycosidic Acids with Unusual Aglycone Units from Convolvulus arvensis. J. Nat. Prod. 2019, 82, 1593-1598. [CrossRef]

15. Muhsinah, A.B.; Alsayari, A.; Thajudeen, K.Y.; Asiri, Y.I.; Nanjaian, M. Simultaneous Estimation of Rutin and Quercetin in Bidens pilosa, Convolvulus arvensis and Neurada procumbens by RP-HPLC. Res. J. Pharm. Technol. 2020, 13, 3305-3310. [CrossRef]

16. Sowemimo, B.O.; Farnsworth, N.R. Phytochemical Investigation of Convolvulus arvensis (Convolvulaceae). J. Pharm. Sc. 1973, 62, 678-679. [CrossRef]

17. Fujiwara, N.; Kobayashi, K. Macrophages in Inflammation. Curr. Drug Targets Inflamm. Allergy 2005, 4, 281-286. [CrossRef]

18. DuBois, R.N.; Abramson, S.B.; Crofford, L.; Gupta, R.A.; Simon, L.S.; van de Putte, L.B.A.; Lipsky, P.E. Cyclooxygenase in Biology and Disease. FASEB J. 1998, 12, 1063-1073. [CrossRef]

19. Korhonen, R.; Lahti, A.; Kankaanranta, H.; Moilanen, E. Nitric Oxide Production and Signaling in Inflammation. CDTIA 2005, 4 , 471-479. [CrossRef] 
20. Netea, M.G.; Balkwill, F.; Chonchol, M.; Cominelli, F.; Donath, M.Y.; Giamarellos-Bourboulis, E.J.; Golenbock, D.; Gresnigt, M.S.; Heneka, M.T.; Hoffman, H.M.; et al. A Guiding Map for Inflammation. Nat. Immunol. 2017, 18, 826-831. [CrossRef]

21. Tasneem, S.; Liu, B.; Li, B.; Choudhary, M.I.; Wang, W. Molecular Pharmacology of Inflammation: Medicinal Plants as AntiInflammatory Agents. Pharmacol. Res. 2019, 139, 126-140. [CrossRef] [PubMed]

22. Wang, M.; Carver, J.J.; Phelan, V.V.; Sanchez, L.M.; Garg, N.; Peng, Y.; Nguyen, D.D.; Watrous, J.; Kapono, C.A.; Luzzatto-Knaan, T.; et al. Sharing and Community Curation of Mass Spectrometry Data with Global Natural Products Social Molecular Networking. Nat. Biotechnol. 2016, 34, 828-837. [CrossRef] [PubMed]

23. Kimura, A.; Kishimoto, T. IL-6: Regulator of Treg/Th17 Balance. Eur. J. Immunol. 2010, 40, 1830-1835. [CrossRef] [PubMed]

24. Neurath, M.F. Cytokines in Inflammatory Bowel Disease. Nat. Rev. Immunol. 2014, 14, 329-342. [CrossRef]

25. Yoshimura, T. The Production of Monocyte Chemoattractant Protein-1 (MCP-1)/CCL2 in Tumor Microenvironments. Cytokine 2017, 98, 71-78. [CrossRef]

26. Umamaheswaran, S.; Dasari, S.K.; Yang, P.; Lutgendorf, S.K.; Sood, A.K. Stress, Inflammation, and Eicosanoids: An Emerging Perspective. Cancer Metastasis Rev. 2018, 37, 203-211. [CrossRef]

27. Salehi, B.; Krochmal-Marczak, B.; Skiba, D.; Patra, J.K.; Das, S.K.; Das, G.; Popović-Djordjević, J.B.; Kostić, A.Ž.; Anil Kumar, N.V.; Tripathi, A.; et al. Convolvulus Plant-A Comprehensive Review from Phytochemical Composition to Pharmacy. Phytother. Res. 2020, 34, 315-328. [CrossRef]

28. Ambriz-Pérez, D.L.; Leyva-López, N.; Gutierrez-Grijalva, E.P.; Heredia, J.B. Phenolic Compounds: Natural Alternative in Inflammation Treatment. A Review. Cogent Food Agric. 2016, 2, 1131412. [CrossRef]

29. Clifford, M.N.; Knight, S.; Kuhnert, N. Discriminating between the Six Isomers of Dicaffeoylquinic Acid by LC-MS ${ }^{n}$. J. Agric. Food Chem. 2005, 53, 3821-3832. [CrossRef]

30. Ablajan, K.; Abliz, Z.; Shang, X.-Y.; He, J.-M.; Zhang, R.-P.; Shi, J.-G. Structural Characterization of Flavonol 3,7-Di-O-Glycosides and Determination of the Glycosylation Position by Using Negative Ion Electrospray Ionization Tandem Mass Spectrometry. J. Mass Spectrom. 2006, 41, 352-360. [CrossRef]

31. Hrichi, S.; Chaabane-Banaoues, R.; Giuffrida, D.; Mangraviti, D.; Oulad el Majdoub, Y.; Rigano, F.; Mondello, L.; Babba, H.; Mighri, Z.; Cacciola, F. Effect of Seasonal Variation on the Chemical Composition and Antioxidant and Antifungal Activities of Convolvulus althaeoides L. Leaf Extracts. Arab. J. Chem. 2020, 13, 5651-5668. [CrossRef]

32. Hassine, M.; Zardi-Berguaoui, A.; Harzallah-Skhiri, F.; Abreu, P.; Jannet, H.B.; Hamza, M.A. Isolation and Structure Elucidation of Secondary Metabolites from the Roots of the Tunisian Convolvulus dorycnium. Chem. Nat. Compd. 2016, 52, 830-833. [CrossRef]

33. Krzaczek, T.; Gogucka-Kocka, A.; Ryn, D. Chromatographical Analysis of Phenolic Compounds in Herb Convolvulus arvensis L. Herba Pol. 2004, 50, 17-22.

34. Yaoya, S.; Kanho, H.; Mikami, Y.; Itani, T.; Umehara, K.; Kuroyanagi, M. Umbelliferone Released from Hairy Root Cultures of Pharbitis nil Treated with Copper Sulfate and Its Subsequent Glucosylation. Biosci. Biotechnol. Biochem. 2004, 68, $1837-1841$. [CrossRef]

35. Takenaka, M.; Nanayama, K.; Isobe, S.; Murata, M. Changes in Caffeic Acid Derivatives in Sweet Potato (Ipomoea batatas L.) during Cooking and Processing. Biosci. Biotechnol. Biochem. 2006, 70, 172-177. [CrossRef]

36. Wang, J.-L.; Hua, Z.; Zhao, B.-Y.; Tang, W.-X.; Zhang, S.-J. Studies on the chemical constituents of Pharbitis purpurea. Zhong Yao Cai 2010, 33, 1571-1574.

37. Belaouira, R.; Marchioni, E.; Benayache, F.; Benayache, S. On-Line Screening and Identification of Polyphenolic Antioxidant Compounds of Convolvulus trabutianus. Nat. Prod. Res. 2020, 34, 1490-1493. [CrossRef]

38. Wang, W.; Xuan, L. New Acyclic Sesquiterpenoid Derivatives and a Monoterpene Disaccharide from Dichondra repens Forst. Phytochem. Lett. 2015, 14, 23-26. [CrossRef]

39. Shahat, A.A.; Abdel-Azim, N.S.; Pieters, L.; Vlietinck, A.J. Flavonoids from Cressa cretica. Pharm. Biol. 2004, 42, 349-352. [CrossRef]

40. Nacef, S.; Jannet, H.B.; Abreu, P.; Mighri, Z. Phenolic Constituents of Convolvulus dorycnium L. Flowers. Phytochem. Lett. 2010, 3 , 66-69. [CrossRef]

41. Zhang, L.; Tu, Z.; Yuan, T.; Wang, H.; Xie, X.; Fu, Z. Antioxidants and $\alpha$-Glucosidase Inhibitors from Ipomoea batatas Leaves Identified by Bioassay-Guided Approach and Structure-Activity Relationships. Food Chem. 2016, 208, 61-67. [CrossRef] [PubMed]

42. Sharma, S.; Ali, A.; Ali, J.; Sahni, J.K.; Baboota, S. Rutin: Therapeutic Potential and Recent Advances in Drug Delivery. Expert Opin. Investig. Drugs 2013, 22, 1063-1079. [CrossRef] [PubMed]

43. Lee, W.; Ku, S.-K.; Bae, J.-S. Barrier Protective Effects of Rutin in LPS-Induced Inflammation In Vitro and In Vivo. Food Chem. Toxicol. 2012, 50, 3048-3055. [CrossRef] [PubMed]

44. Naveed, M.; Hejazi, V.; Abbas, M.; Kamboh, A.A.; Khan, G.J.; Shumzaid, M.; Ahmad, F.; Babazadeh, D.; FangFang, X.; Modarresi-Ghazani, F.; et al. Chlorogenic Acid (CGA): A Pharmacological Review and Call for Further Research. Biomed. Pharmacother. 2018, 97, 67-74. [CrossRef]

45. Hwang, S.J.; Kim, Y.-W.; Park, Y.; Lee, H.-J.; Kim, K.-W. Anti-Inflammatory Effects of Chlorogenic Acid in LipopolysaccharideStimulated RAW 264.7 Cells. Inflamm. Res. 2014, 63, 81-90. [CrossRef]

46. Jiang, Y.; Yu, L.; Wang, M.-H. N-Trans-Feruloyltyramine Inhibits LPS-Induced NO and PGE 2 Production in RAW 264.7 Macrophages: Involvement of AP-1 and MAP Kinase Signalling Pathways. Chem. Biol. Interact. 2015, 235, 56-62. [CrossRef] 
47. Le, T.B.; Beaufay, C.; Nghiem, D.T.; Mingeot-Leclercq, M.-P.; Quetin-Leclercq, J. In Vitro Anti-Leishmanial Activity of Essential Oils Extracted from Vietnamese Plants. Molecules 2017, 22, 1071. [CrossRef]

48. Masquelier, J.; Alhouayek, M.; Terrasi, R.; Bottemanne, P.; Paquot, A.; Muccioli, G.G. Lysophosphatidylinositols in Inflammation and Macrophage Activation: Altered Levels and Anti-Inflammatory Effects. Biochim. Biophys. Acta Mol. Cell Biol. Lipids 2018, 1863, 1458-1468. [CrossRef]

49. Alhouayek, M.; Bottemanne, P.; Makriyannis, A.; Muccioli, G.G. N-Acylethanolamine-Hydrolyzing Acid Amidase and Fatty Acid Amide Hydrolase Inhibition Differentially Affect $N$-Acylethanolamine Levels and Macrophage Activation. Biochim. Biophys Acta. Mol. Cell Biol. Lipids 2017, 1862, 474-484. [CrossRef]

50. Alhouayek, M.; Masquelier, J.; Cani, P.D.; Lambert, D.M.; Muccioli, G.G. Implication of the Anti-Inflammatory Bioactive Lipid Prostaglandin D2-Glycerol Ester in the Control of Macrophage Activation and Inflammation by ABHD6. Proc. Natl. Acad. Sci. USA 2013, 110, 17558-17563. [CrossRef]

51. Pluskal, T.; Castillo, S.; Villar-Briones, A.; Orešič, M. MZmine 2: Modular Framework for Processing, Visualizing, and Analyzing Mass Spectrometry-Based Molecular Profile Data. BMC Bioinform. 2010, 11, 395. [CrossRef] [PubMed]

52. Shannon, P.; Markiel, A.; Ozier, O.; Baliga, N.S.; Wang, J.T.; Ramage, D.; Amin, N.; Schwikowski, B.; Ideker, T. Cytoscape: A Software Environment for Integrated Models of Biomolecular Interaction Networks. Genome Res. 2003, 13, 2498-2504. [CrossRef] [PubMed] 